\title{
Isotropic Material Design
}

\author{
S. Czarnecki \\ Department of Structural Mechanics and Computer Aided Engineering \\ Warsaw University of Technology \\ Al. Armii Ludowej 16, 00-637 Warsaw, Poland \\ E-mail: s.czarnecki@il.pw.edu.pl
}

Received: 24 October 2014; revised: 19 April 2015; accepted: 20 April 2015; published online: 02 June 2015

\begin{abstract}
The paper deals with optimal distribution of the bulk and shear moduli minimizing the compliance of an inhomogeneous isotropic elastic 3D body transmitting a given surface loading to a given support. The isoperimetric condition is expressed by the integral of the trace of the Hooke tensor being a linear combination of both moduli. The problem thus formulated is reduced to an auxiliary 3D problem of minimization of a certain stress functional over the stresses being statically admissible. The integrand of the auxiliary functional is a linear combination of the absolute value of the trace and norm of the deviator of the stress field. Thus the integrand is of linear growth. The auxiliary problem is solved numerically by introducing element-wise polynomial approximations of the components of the trial stress fields and imposing satisfaction of the variational equilibrium equations. The under-determinate system of these equations is solved numerically thus reducing the auxiliary problem to an unconstrained problem of nonlinear programming.
\end{abstract}

Key words: isotropic elasticity, topology optimization, free material design, compliance minimization

\section{INTRODUCTION}

The problem of optimal designing of structural topology encompasses two main problems: a) optimum layout of two or several materials (e.g. homogeneous isotropic materials) within a given design domain, or: b) optimum distribution of selected characteristics of the material properties. In linear elasticity problems the central functional undergoing minimization in structural optimization is the compliance of the whole structure, or the work of given loading done on the displacements caused by the same loading. In problem (a) the amount of one of the materials can be chosen as the isoperimetric condition. In problem (b) the elastic moduli $C_{i j k l}$ are design variables; the isoperimetric condition should be expressed in terms of these moduli. A rational choice is to assume that an integral of a scalar and invariant function of the moduli is fixed. This scalar function is usually chosen as the trace of the Hooke tensor.
Problems (a) and (b) are the core of the monographs [1-3]. Problem (a) includes also the shape forming, if one of two materials is a void. In each case problem (a) needs relaxation. The relaxed formulation concerning the two material case has been thoroughly discussed in the mentioned references. The relaxation of the three material problem (or two materials surrounded by voids) is the subject of the contemporary research, see [4].

Problem (b) is called Free Material Design (FMD) (or Free Material Optimization), see [1], if the sought Hooke tensor is subject only to usual symmetry conditions and conditions of semi-positive definiteness. Thus the free material approach does not introduce constraints on the anisotropy. Some authors require positive definiteness to prevent from possible instabilities, which introduces a new parameter into the design. In case of a single load condition the FMD problem has been solved by Bendsøe et al. [5]. The optimal tensor $C$ has only one non-zero eigenvalue. The anisotropy directions are collinear with principal directions of the stress state, the 
principal directions of strain coincide with the latter. In spite of the mentioned degeneracy the static problem is solvable for the given loading, as proved in Werner's thesis [6] and in Haslinger et al. [7]. The stress based approach by Czarnecki and Lewiński [8-10] shows that the FMD problem reduces to a seemingly simple, but mathematically subtle problem: minimize a functional of an integrand of linear growth. This formulation explains why the FMD problem solves simultaneously the shape optimization problem: the minimizer of the mentioned variational problem has a bounded effective domain whose boundary determines the shape of the optimal structure, see Fig. 3 in [9]. Thus the solution cuts out the optimal structure from the given design domain, this solution being made from the optimal anisotropic and inhomogeneous material.

To prevent from degeneracy of the optimal Hooke tensor one can take into account more than one load condition. To construct the Pareto front one should minimize the convex combination of the compliances corresponding to the load conditions, see [10], Secs. 2, 3, 6. It turns out that only six independent load conditions result in nonzero values of all eigenvalues of the optimal elasticity tensor in 3D. In 2D case three load conditions suffice to assure the same property.

Alternatively, one can assume a priori that the design should be characterized at each point by some material symmetries. The strongest assumption of this kind: isotropy is the subject of the present paper. Thus the aim of the study is to pose and solve the problem of minimization of the compliance of an inhomogeneous and isotropic body subject to a single load condition; the design variables are Kelvin and Kirchhoff moduli, also named bulk and shear moduli and denoted by $k(x)$ and $\mu(x), x$ being a point in a domain $\Omega$. The tractions on a part of the boundary are prescribed. The tractions should be transmitted to the other given part of the boundary, called a support. By analogy with the free material design proposed in [5] we impose the isoperimetric condition:

$$
\int_{\Omega} \operatorname{tr} C \mathrm{~d} x=\Lambda
$$

where, due to isotropy $\operatorname{tr} C=3 k+10 \mu$. A proof will be delivered that the solution to the problem above is rationally posed, thus leading to explicit formulae for the optimal moduli $k^{*}, \mu^{*}$. The problem above will be referred to as the Isotropic Material Design (IMD).

The problem above is free of any additional conditions; the moduli are not viewed as bounded; the integrability condition due to (I.1) is the only assumed regularity condition. Due to this setting the moduli of the optimal material can attain extreme admissible values.

The isotropic characterization of the solutions of the FMD problem in its original setting [5] can be imposed by reconstruction of an isotropic tensor $\boldsymbol{H}$ which at each point of the body lies as close as possible to the optimal tensor $C^{*}$ of anisotropic structure, in the metric defined by the Frobenius norm or other norms, e.g. the logarithmic norm discussed in [11]. The Frobenius norm is justified by the condition of preserving the value of the trace of both tensors. It turns out that the isotropic moduli $k$ and $\mu$ constructed in this manner have much in common with their counterparts constructed by the IMD method. Although we have no mathematical proof that they coincide, the computations suggests that this is the case.

\section{FORMULATION OF AN OPTIMAL DESIGN}

\section{1. Isotropic material design}

The aim of this section is to propose an algorithm of designing optimal distribution of isotropic characteristics to minimize the compliance. The method will be called Isotropic Material Design (IMD) and is put forward here as a modification of the Free Material Design (FMD).

Consider a 3D elastic body $\Omega$ with $|\Omega|$ denoting its volume. The body is fixed at the boundary surface $\Gamma_{2}$. The $\Gamma_{1}$ segment of the boundary is subject to the tractions of intensity $\boldsymbol{T}$. The domain $\Omega$ is parameterized by the Cartesian coordinates $x_{1}, x_{2}, x_{3}$ with the orthonormal basis $\left\{\boldsymbol{e}_{1}, \boldsymbol{e}_{2}, \boldsymbol{e}_{3}\right\}$; $x=\left(x_{1}, x_{2}, x_{3}\right)$ is a point in $\Omega$. The unknown displacement field $\boldsymbol{u}=\left(u_{1}, u_{2}, u_{3}\right)$ and the virtual displacement field $\boldsymbol{v}=\left(v_{1}, v_{2}, v_{3}\right)$ are kinematically admissible if they vanish on $\Gamma_{2}$; the regularity conditions, implicitly present in the definition of the space $V(\Omega)$ of the kinematically admissible displacement fields, will not be discussed. Let $S^{3}$ be the set of symmetric tensors of the $2^{\text {nd }}$ rank in 3D Euclidean space (equivalently, symmetric matrices of order 3 ). The strains constitute a tensor $\varepsilon=\left(\varepsilon_{i j}\right) \in S^{3}$ of components $\varepsilon_{i j}(\boldsymbol{u})$ determined by the displacement vector:

$$
\varepsilon_{i j}(\boldsymbol{u})=\left(u_{i, j}+u_{j, i}\right) / 2
$$

where $(\cdot)_{,}=\partial / \partial x_{i}, \quad i, j=1,2,3$. The stresses $\boldsymbol{\sigma}=$ $\left(\sigma_{i j}\right) \in S^{3}$ are linked with strains by the Hooke law

$$
\boldsymbol{\sigma}(\boldsymbol{u})=\boldsymbol{C} \varepsilon(\boldsymbol{u})
$$

where $C=\left(C_{i j k l}\right)$ is the $4^{\text {th }}$ rank Hooke's tensor of known symmetries in 3D Euclidean space. Let $S^{6}$ be the set of symmetric matrices of order 6 . If the stress and strain tensors are replaced by vectors according to the

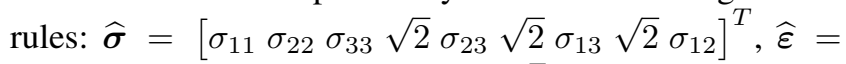

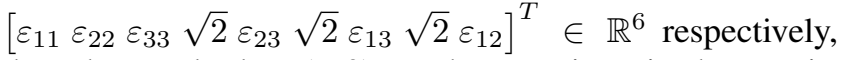
then the Hooke law (II.2) can be re-written in the matrix form

$$
\widehat{\sigma}=\widehat{C} \widehat{\varepsilon}
$$

where the matrix of elastic moduli $\widehat{C}=\left(\widehat{C}_{i j}\right) \in S^{6}$ reads 


$$
\widehat{\boldsymbol{C}}=\left[\begin{array}{llllll}
C_{1111} & C_{1122} & C_{1133} & \sqrt{2} C_{1123} & \sqrt{2} C_{1113} & \sqrt{2} C_{1112} \\
& C_{2222} & C_{2233} & \sqrt{2} C_{2223} & \sqrt{2} C_{2213} & \sqrt{2} C_{2212} \\
& & C_{3333} & \sqrt{2} C_{3323} & \sqrt{2} C_{3313} & \sqrt{2} C_{3312} \\
& & & 2 C_{2323} & 2 C_{2313} & 2 C_{2312} \\
& \text { sym } & & & 2 C_{1313} & 2 C_{1312} \\
& & & & & 2 C_{1212}
\end{array}\right] .
$$

The unique algebraic properties of the representation (II.3), (II.4) are cleared up in e.g. [11]. Almost everywhere in $\Omega$ the components of tensor $C$ satisfy the positivity condition $C_{i j k l} \varepsilon_{i j} \varepsilon_{k l} \geq c \varepsilon_{i j} \varepsilon_{i j}$ for some $c>0$, the summation convention over indices $i, j, k, l$ being adopted. Moreover, we assume that $C_{i j k l} \in L^{\infty}(\Omega)$. Tensor fields $C$ satisfying the conditions above constitute a set $H(\Omega)$ of admissible fields of Hooke's tensors. The test stress field $\tau=\left(\tau_{i j}\right) \in S^{3}$ at each point $x \in \Omega$ is said to be statically admissible if the following variational equation holds

$$
\forall \boldsymbol{v} \in V(\Omega) \quad \int_{\Omega} \boldsymbol{\tau} \cdot \delta \varepsilon \mathrm{d} x=\int_{\Gamma_{1}} \boldsymbol{T} \cdot \boldsymbol{v} \mathrm{d} a
$$

where $\boldsymbol{\tau} \cdot \delta \boldsymbol{\varepsilon}=\boldsymbol{\tau} \cdot \boldsymbol{\varepsilon}(\boldsymbol{v})=\tau_{i j} \varepsilon_{i j}$ and $\boldsymbol{T} \cdot \boldsymbol{v}=T_{i} v_{i}$; the dots mean the scalar products in $S^{3}$ and $\mathbb{R}^{3}$, respectively. The linear form on $V(\Omega), f=f(\boldsymbol{v})=\int_{\Gamma_{1}} \boldsymbol{T} \cdot \boldsymbol{v} \mathrm{d} a$ is called the virtual work of the given loading. The norm defined by the scalar product" . " in $S^{3}$ is denoted by $\|\cdot\|$, i.e. $\|\boldsymbol{\tau}\|=(\boldsymbol{\tau} \cdot \boldsymbol{\tau})^{1 / 2}$. The set of fields $\boldsymbol{\tau} \in S^{3}$ satisfying (II.5) is denoted by $\Sigma(\Omega)$. If $\boldsymbol{u} \in V(\Omega)$, while $\boldsymbol{\sigma}$ is linked with $\boldsymbol{u}$ by (II.2), satisfies (II.5), then such a field $u$ is a solution of the given equilibrium problem. Let us write $\boldsymbol{u}=\boldsymbol{u}(\boldsymbol{C})$ to stress that $\boldsymbol{u}$ is determined by tensor field $\boldsymbol{C}$. The functional

$$
\wp: H(\Omega) \rightarrow \mathbb{R}, \quad \wp(\boldsymbol{C})=f(\boldsymbol{u}(\boldsymbol{C}))
$$

is called the compliance. The Castigliano theorem provides the formula

$$
\forall \boldsymbol{C} \in H(\Omega) \quad \wp(\boldsymbol{C})=\min _{\boldsymbol{\tau} \in \Sigma(\Omega)} \int_{\Omega} \boldsymbol{\tau} \cdot \boldsymbol{C}^{-1} \boldsymbol{\tau} \mathrm{d} x
$$

see e.g. [12]. The assumption $C \in H(\Omega)$ is sufficient for (II.7) to hold. Assume that all components of tensor fields $C \in H(\Omega)$ are design variables. Let us define functional

$$
\Re: H(\Omega) \rightarrow \mathbb{R}, \quad \Re(\boldsymbol{C})=\int_{\Omega} \operatorname{tr} \boldsymbol{C} \mathrm{d} x-\Lambda,
$$

where

$$
\operatorname{tr} \boldsymbol{C}=C_{i j i j}=\widehat{C}_{i i}=\operatorname{tr} \widehat{\boldsymbol{C}}, \Lambda=E_{0}|\Omega|
$$

and $E_{0}$ is a given elastic modulus. We assume that $\Re(\boldsymbol{C})=0$ which means that the design variables $C \in H(\Omega)$ are additionally subject to the resource constraints that could be interpreted e.g. as the cost of the design, the integrand (II.9) being viewed as a unit cost of the material and $\Lambda$ being viewed as the entire cost of the design proportional to the value of $E_{0}$. The problem: find a tensor field $C^{*} \in H(\Omega)$ such that

$$
\wp^{*}=\wp\left(\boldsymbol{C}^{*}\right)=\min _{\boldsymbol{C} \in H(\Omega)} \wp(\boldsymbol{C}), \Re\left(\boldsymbol{C}^{*}\right)=0
$$

is usually called the Free Material Design (FMD) or Free Material Optimization (FMO) problem. Let us define the set

$$
H^{\Re}(\Omega)=\{\boldsymbol{C} \in H(\Omega) \mid \Re(\boldsymbol{C})=0\}
$$

and insert the formula (II.7) into (II.10) $)_{1}$. Then, interchanging the sequence of operators min (let us note that the set $\Sigma(\Omega)$ of the statically admissible stresses does not depend on the design variables $C \in H^{\Re}(\Omega)$ ), we can replace the problem (II.10) with the following one

$$
\wp^{*}=\wp\left(\boldsymbol{C}^{*}\right)=\min _{\boldsymbol{\tau} \in \Sigma(\Omega)} \min _{\boldsymbol{C} \in H^{\Re}(\Omega)} \int_{\Omega} \boldsymbol{\tau} \cdot \boldsymbol{C}^{-1} \boldsymbol{\tau} \mathrm{d} x .
$$

To have in view finding the solution of the "inner" minimization problem $\min _{\boldsymbol{C} \in H^{\Re}(\Omega)} \int_{\Omega} \boldsymbol{\tau} \cdot \boldsymbol{C}^{-1} \boldsymbol{\tau} \mathrm{d} x$ for arbitrary but fixed admissible stress field $\tau \in \Sigma(\Omega)$, we define the functional

$$
J_{\boldsymbol{\tau}}: H(\Omega) \rightarrow \mathbb{R}, \quad J_{\boldsymbol{\tau}}(\boldsymbol{C})=\int_{\Omega} \boldsymbol{\tau} \cdot \boldsymbol{C}^{-1} \boldsymbol{\tau} \mathrm{d} x
$$

and formulate the following (auxiliary) problem: find a tensor field $\boldsymbol{C}_{\boldsymbol{\tau}}^{*} \in H(\Omega)$ such that

$$
J_{\boldsymbol{\tau}}^{*}=J_{\boldsymbol{\tau}}\left(\boldsymbol{C}_{\boldsymbol{\tau}}^{*}\right)=\min _{\boldsymbol{C} \in H(\Omega)} J_{\boldsymbol{\tau}}(\boldsymbol{C}), \Re\left(\boldsymbol{C}_{\boldsymbol{\tau}}^{*}\right)=0 .
$$

To shorten notation, the above problem can be equivalently written as

$$
J_{\boldsymbol{\tau}}^{\Re *}=J_{\boldsymbol{\tau}}^{\Re}\left(\boldsymbol{C}_{\boldsymbol{\tau}}^{*}\right)=\min _{\boldsymbol{C} \in H^{\Re}(\Omega)} J_{\boldsymbol{\tau}}^{\Re}(\boldsymbol{C}),
$$

where $J_{\tau}^{\Re}$ is a truncation of the functional $J_{\tau}$ to the domain $H^{\Re}(\Omega)$.

Our aim is to construct optimal isotropy of varying characteristics. Thus we impose now the isotropy constraints on the Hooke tensors $C$ in (II.12). Let us write down the spectral decomposition of the arbitrary non-homogeneous, isotropic Hooke tensor $\boldsymbol{H}$, see [11]

$$
\boldsymbol{H}=3 k \boldsymbol{\Lambda}_{1}+2 \mu \boldsymbol{\Lambda}_{2}
$$


where $\boldsymbol{\Lambda}_{1}=\frac{1}{3} \delta_{i j} \delta_{k l} \boldsymbol{e}_{i} \otimes \boldsymbol{e}_{j} \otimes \boldsymbol{e}_{k} \otimes \boldsymbol{e}_{l}, \boldsymbol{\Lambda}_{2}=$ $\frac{1}{2}\left(\delta_{i k} \delta_{j l}+\delta_{i l} \delta_{j k}\right) \boldsymbol{e}_{i} \otimes \boldsymbol{e}_{j} \otimes \boldsymbol{e}_{k} \otimes \boldsymbol{e}_{l}-\boldsymbol{\Lambda}_{1}$. The quantities $k$ and $\mu$ are bulk and shear moduli linked with Young's modulus $E$ and Poisson's ratio $\nu$ by

$$
\begin{aligned}
& k=\frac{E}{3(1-2 \nu)}>0, \mu=\frac{E}{2(1+\nu)}>0, \\
& E=\frac{9 k \mu}{3 k+\mu}>0,-1<\nu=\frac{3 k-2 \mu}{2(3 k+\mu)}<\frac{1}{2} .
\end{aligned}
$$

The bulk and shear moduli will be the design variables. The fourth order tensors $\Lambda_{1}, \Lambda_{2}$ are mutually orthogonal projectors having the well known properties

$$
\boldsymbol{\Lambda}_{1} \boldsymbol{\Lambda}_{1}=\boldsymbol{\Lambda}_{1}, \boldsymbol{\Lambda}_{1} \boldsymbol{\Lambda}_{2}=\boldsymbol{\Lambda}_{2} \boldsymbol{\Lambda}_{1}=0, \boldsymbol{\Lambda}_{2} \boldsymbol{\Lambda}_{2}=\boldsymbol{\Lambda}_{2}
$$

Let us compute the integrand in (II.13) by using representation (II.16)

$$
\boldsymbol{\tau} \cdot \boldsymbol{H}^{-1} \boldsymbol{\tau}=\frac{1}{3 k} \boldsymbol{\tau} \cdot \boldsymbol{\Lambda}_{1} \boldsymbol{\tau}+\frac{1}{2 \mu} \boldsymbol{\tau} \cdot \boldsymbol{\Lambda}_{2} \boldsymbol{\tau}
$$

and introduce a counterpart of the (II.13)

$$
\begin{aligned}
& J_{\boldsymbol{\tau}}: L(\Omega) \times L(\Omega) \rightarrow \mathbb{R} \\
& J_{\boldsymbol{\tau}}(k, \mu)=\int_{\Omega}\left(\frac{1}{3 k} \boldsymbol{\tau} \cdot \boldsymbol{\Lambda}_{1} \boldsymbol{\tau}+\frac{1}{2 \mu} \boldsymbol{\tau} \cdot \boldsymbol{\Lambda}_{2} \boldsymbol{\tau}\right) \mathrm{d} x
\end{aligned}
$$

where $L(\Omega)$ represents the space of integrable functions (with integrable inverses) defined on the domain $\Omega$ and assuming positive real values. Similarly, we redefine the functional (II.8) as

$\Re: L(\Omega) \times L(\Omega) \rightarrow \mathbb{R}, \Re(k, \mu)=\int_{\Omega}(3 k+10 \mu) \mathrm{d} x-\Lambda$.

The definition (II.21) results immediately from the formula

$$
\operatorname{tr} \boldsymbol{C}=\sum_{K=1}^{6} \lambda_{K}
$$

for anisotropic Hooke tensor $\boldsymbol{C}$, where its eigenvalues (Kelvin moduli) $\lambda_{K}, K=1,2, \ldots, 6$ for the isotropic bodies are:

$$
\lambda_{1}=3 k, \lambda_{2}=\ldots=\lambda_{6}=2 \mu
$$

(see e.g. (3.28), (3.29) in [9]). The problem (II.14) is now replaced by: find $\left(k_{\boldsymbol{\tau}}^{*}, \mu_{\boldsymbol{\tau}}^{*}\right) \in L(\Omega) \times L(\Omega)$ such that

$$
\begin{aligned}
& J_{\boldsymbol{\tau}}^{*}=J_{\boldsymbol{\tau}}\left(k_{\boldsymbol{\tau}}^{*}, \mu_{\boldsymbol{\tau}}^{*}\right)= \\
= & \min _{(k, \mu) \in L(\Omega) \times L(\Omega)} \int_{\Omega}\left(\frac{1}{3 k} \boldsymbol{\tau} \cdot \boldsymbol{\Lambda}_{1} \boldsymbol{\tau}+\frac{1}{2 \mu} \boldsymbol{\tau} \cdot \boldsymbol{\Lambda}_{2} \boldsymbol{\tau}\right) \mathrm{d} x, \\
& \int_{\Omega}\left(3 k_{\boldsymbol{\tau}}^{*}+10 \mu_{\boldsymbol{\tau}}^{*}\right) \mathrm{d} x-\Lambda=0 .
\end{aligned}
$$

One of possible ways of dealing with the constrained problem (II.24) is the method of Lagrange multipliers. Let us introduce the Lagrangian variable $\eta$ and the Lagrange functional

$$
\begin{aligned}
& \Im_{\boldsymbol{\tau}}: L(\Omega) \times L(\Omega) \times \mathbb{R}^{*} \rightarrow \mathbb{R}, \\
& \Im_{\boldsymbol{\tau}}(k, \mu, \eta)=\int_{\Omega}\left(\frac{V_{1}^{\boldsymbol{\tau}}}{k}+\frac{V_{2}^{\boldsymbol{\tau}}}{\mu}\right) \mathrm{d} x \\
& +\eta\left(\int_{\Omega}(3 k+10 \mu) \mathrm{d} x-\Lambda\right) .
\end{aligned}
$$

The three necessary local optimality conditions read:

$$
\frac{\partial \Im_{\boldsymbol{\tau}}}{\partial k}(k, \mu, \eta)=0, \frac{\partial \Im_{\boldsymbol{\tau}}}{\partial \mu}(k, \mu, \eta)=0, \frac{\partial \Im_{\boldsymbol{\tau}}}{\partial \eta}(k, \mu, \eta)=0,
$$

where

$$
\begin{aligned}
V_{1}^{\boldsymbol{\tau}} & =\frac{1}{3} \boldsymbol{\tau} \cdot \boldsymbol{\Lambda}_{1} \boldsymbol{\tau}=\frac{1}{9}(\operatorname{tr} \boldsymbol{\tau})^{2} \\
V_{2}^{\boldsymbol{\tau}} & =\frac{1}{2} \boldsymbol{\tau} \cdot \boldsymbol{\Lambda}_{2} \boldsymbol{\tau}=\frac{1}{2}\left(\boldsymbol{\tau} \cdot \boldsymbol{\tau}-\frac{1}{3}(\operatorname{tr} \boldsymbol{\tau})^{2}\right) .
\end{aligned}
$$

Upon computing the first partial derivatives and applying the lemma of Du Bois-Reymond we get the three equations with two unknown scalar fields $k, \mu$ and a Lagrange multiplier $\eta$ :

$-\frac{V_{1}^{\boldsymbol{\tau}}}{k^{2}}+3 \eta=0,-\frac{V_{2}^{\boldsymbol{\tau}}}{\mu^{2}}+10 \eta=0, \int_{\Omega}(3 k+10 \mu) \mathrm{d} x=\Lambda$.

The solution of the three above equations leads to the explicit formulae of the optimal fields $k_{\tau}^{*}, \mu_{\boldsymbol{\tau}}^{*} \in L(\Omega)$

$$
\begin{aligned}
& k_{\boldsymbol{\tau}}^{*}=\sqrt{10} \Lambda \frac{\sqrt{V_{1}^{\boldsymbol{\tau}}}}{\int_{\Omega}\left(3 \sqrt{10} \sqrt{V_{1}^{\boldsymbol{\tau}}}+10 \sqrt{3} \sqrt{V_{2}^{\boldsymbol{\tau}}}\right) \mathrm{d} x}, \\
& \mu_{\boldsymbol{\tau}}^{*}=\sqrt{3} \Lambda \frac{\sqrt{V_{2}^{\boldsymbol{\tau}}}}{\int_{\Omega}\left(3 \sqrt{10} \sqrt{V_{1}^{\boldsymbol{\tau}}}+10 \sqrt{3} \sqrt{V_{2}^{\boldsymbol{\tau}}}\right) \mathrm{d} x}
\end{aligned}
$$

and provides the optimal value $J_{\tau}^{*}$ of the functional (II.20)

$$
J_{\boldsymbol{\tau}}^{*}=\frac{1}{30 \Lambda}\left(\int_{\Omega}\left(3 \sqrt{10} \sqrt{V_{1}^{\boldsymbol{\tau}}}+10 \sqrt{3} \sqrt{V_{2}^{\boldsymbol{\tau}}}\right) \mathrm{d} x\right)^{2} .
$$

Let us remind that the minimizers $k_{\tau}^{*}, \mu_{\tau}^{*}$ together with the optimal value $J_{\tau}^{*}$ of the functional (II.20) depend on still unknown statically admissible stress field $\tau \in \Sigma(\Omega)$, i.e. $k_{\boldsymbol{\tau}}^{*}=k_{\boldsymbol{\tau}}^{*}(\boldsymbol{\tau}), \mu_{\boldsymbol{\tau}}^{*}=\mu_{\boldsymbol{\tau}}^{*}(\boldsymbol{\tau}), J_{\boldsymbol{\tau}}^{*}=J_{\boldsymbol{\tau}}^{*}(\boldsymbol{\tau})$. In other words, we have found at this stage of the analysis only the solution of the "inner" minimization problem (II.14). In the last step, we show how to numerically find the solution of the original problem (II.12), i.e. we show how to numerically find the statically admissible minimizer $\tau^{*} \in \Sigma(\Omega)$. Let us note that the optimal compliance (II.10) is expressed by

$$
\wp^{*}=\frac{1}{30 \Lambda}\left(S^{*}\right)^{2}
$$




$$
S^{*}=\min _{\tau \in \Sigma(\Omega)} \int_{\Omega} \Pi(\tau(x)) \mathrm{d} x
$$

where the integrand $\Pi: S^{3} \rightarrow \mathbb{R}$ is defined as

$$
\begin{aligned}
& \Pi(\boldsymbol{\tau})=t|\operatorname{tr} \boldsymbol{\tau}|+d \sqrt{\boldsymbol{\tau} \cdot \boldsymbol{\tau}-\frac{1}{3}(\operatorname{tr} \boldsymbol{\tau})^{2}}= \\
= & t|\operatorname{tr} \boldsymbol{\tau}|+d\|\operatorname{dev} \boldsymbol{\tau}\|
\end{aligned}
$$

where dev $\boldsymbol{\tau}=\boldsymbol{\tau}-\frac{1}{3}(\operatorname{tr} \boldsymbol{\tau}) \boldsymbol{I} \in S^{3}$ is the deviator of the stress tensor $\tau, \boldsymbol{I}$ being the unit tensor in $S^{3}$ and $t=\sqrt{10}, d=5 \sqrt{6}$. Let $\tau^{*} \in \Sigma(\Omega)$ represents the minimizer of (II.31b). Having $\tau^{*}$ one can compute the optimal bulk and shear moduli by (II.29). The optimal elastic isotropic Hooke tensor assumes the form

$$
\boldsymbol{H}^{*}=3 k^{*} \boldsymbol{\Lambda}_{1}+2 \mu^{*} \boldsymbol{\Lambda}_{2}
$$

and one can calculate the optimal compliance (II.31a) as

$$
\wp^{*}=\wp\left(\boldsymbol{H}^{*}\right)=J^{*},
$$

where

$$
J^{*}=J_{\boldsymbol{\tau}^{*}}^{*} \quad, \quad k^{*}=k_{\boldsymbol{\tau}^{*}}^{*} \quad, \quad \mu^{*}=\mu_{\boldsymbol{\tau}^{*}}^{*}
$$

According to (II.1), the optimal solutions (II.35) 2,3 , (II.33) determine the optimal stress components via the strain tensor $\varepsilon=\varepsilon(\boldsymbol{u})$

$$
\begin{aligned}
& \boldsymbol{\sigma}^{*}=\boldsymbol{\sigma}^{*}(\boldsymbol{u})=\boldsymbol{H}^{*} \boldsymbol{\varepsilon}(\boldsymbol{u})= \\
= & \frac{\Lambda\left(\sqrt{10 V_{1}^{\boldsymbol{\tau}^{*}}}(\operatorname{tr} \boldsymbol{\varepsilon}(\boldsymbol{u})) \boldsymbol{I}+2 \sqrt{3 V_{2}^{\boldsymbol{\tau}^{*}}} \operatorname{dev} \boldsymbol{\varepsilon}(\boldsymbol{u})\right)}{\int_{\Omega}\left(3 \sqrt{10} \sqrt{V_{1}^{\boldsymbol{\tau}^{*}}}+10 \sqrt{3} \sqrt{V_{2}^{\boldsymbol{\tau}^{*}}}\right) \mathrm{d} x}
\end{aligned}
$$

and the unknown vector field of the displacements $\boldsymbol{u}=\boldsymbol{u}(x)$, $x \in \Omega$ can be found from the variational equilibrium equation (II.5), i.e.

$$
\forall \boldsymbol{v} \in V(\Omega) \quad a_{\boldsymbol{H}^{*}}(\boldsymbol{u}, \boldsymbol{v})=f(\boldsymbol{v})
$$

where

$$
a_{\boldsymbol{H}^{*}}(\boldsymbol{u}, \boldsymbol{v})=\int_{\Omega} \boldsymbol{H}^{*} \varepsilon(\boldsymbol{u}) \cdot \boldsymbol{\varepsilon}(\boldsymbol{v}) \mathrm{d} x .
$$

Remark II.1. It can be shown that the problem (II.37) is well posed and has unique solution $\boldsymbol{u} \in V(\Omega)$ always if $k^{*}>0$ or $\mu^{*}>0$. In other cases, a much more mathematically rigorous analysis has to be discussed, which is out of the scope of the paper (in the case of the non-homogeneous, optimal anisotropic design, see discussion on pp. 227-229 in [9]).

\section{2. Design of optimal anisotropy}

Let us briefly recapitulate the main results of the FMD problem in its stress-based setting, cf. $[9,10]$. Let $\tau^{*}$ be the minimizer of the problem:

$$
\min _{\boldsymbol{\tau} \in \Sigma(\Omega)} \int_{\Omega}\|\boldsymbol{\tau}\| \mathrm{d} x .
$$

The optimal anisotropic tensor is expressed by

$$
\boldsymbol{C}^{*}=\frac{\lambda_{1}^{*}}{\left\|\boldsymbol{\tau}^{*}\right\|^{2}} \boldsymbol{\tau}^{*} \otimes \boldsymbol{\tau}^{*}
$$

where the optimal values of Kelvin moduli are

$$
\lambda_{1}^{*}=\Lambda \frac{\left\|\boldsymbol{\tau}^{*}\right\|}{\int_{\Omega}\left\|\boldsymbol{\tau}^{*}\right\| \mathrm{d} x} \quad, \quad \lambda_{2}^{*}=\lambda_{3}^{*}=\lambda_{4}^{*}=\lambda_{5}^{*}=\lambda_{6}^{*}=0
$$

and the optimal compliance of the body is then equal

$$
\wp^{*}=\wp\left(\boldsymbol{C}^{*}\right)=J^{*}
$$

with $J^{*}$ expressed by

$$
J^{*}=J_{\tau^{*}}^{*}=\frac{1}{\Lambda}\left(\int_{\Omega}\left\|\tau^{*}\right\| \mathrm{d} x\right)^{2} .
$$

Similarly as in (II.36) the optimal stress components could be calculated via the formulae

$$
\boldsymbol{\sigma}^{*}=\boldsymbol{\sigma}^{*}(\boldsymbol{u})=\frac{\lambda_{1}^{*}\left(\boldsymbol{\tau}^{*} \cdot \boldsymbol{\varepsilon}(\boldsymbol{u})\right)}{\left\|\boldsymbol{\tau}^{*}\right\|^{2}} \boldsymbol{\tau}^{*}
$$

where the unknown vector field of the displacements $\boldsymbol{u}=$ $\boldsymbol{u}(x), x \in \Omega$ can be found from the variational equilibrium equation (II.5), i.e.

$$
\begin{aligned}
& \forall \boldsymbol{v} \in V(\Omega) \quad a_{\boldsymbol{C}^{*}}(\boldsymbol{u}, \boldsymbol{v})=f(\boldsymbol{v}), \\
& a_{\boldsymbol{C}^{*}}(\boldsymbol{u}, \boldsymbol{v})=\int_{\Omega} \boldsymbol{\sigma}^{*}(\boldsymbol{u}) \cdot \boldsymbol{\varepsilon}(\boldsymbol{v}) \mathrm{d} x .
\end{aligned}
$$

Remark II.2. A similar discussion as in Remark II.1 is necessary to motivate the correctness of the mathematical definition of the displacement based problem (II.44) (see discussion on pp. 227-229 in [9] and [10]).

\section{THE STRESS-BASED NUMERICAL APPROACH TO SOLVING THE IMD PROBLEM}

We will present the newly developed scheme of construction of statically admissible stress fields defined element-wise, along with the optimizer solving the minimization problem. The numerical approach starts from division of the design domain $\Omega$ into 8-node, cubic, conforming, isoparametric finite elements with shape functions

$$
\begin{aligned}
N_{i}(\xi)= & \frac{1}{8}\left(1 \pm \xi_{1} \pm \xi_{2} \pm \xi_{3} \pm \xi_{1} \xi_{2}\right. \\
& \left. \pm \xi_{2} \xi_{3} \pm \xi_{1} \xi_{3} \pm \xi_{1} \xi_{2} \xi_{3}\right)
\end{aligned}(i=1,2, \ldots, 8)
$$


defined on the master element interpolating the nine stress fields

$$
\begin{aligned}
& \tau_{11}(x)=N_{1}(\xi) \tau_{1}+N_{2}(\xi) \tau_{7}+\ldots+N_{8}(\xi) \tau_{43} \\
& \tau_{22}(x)=N_{1}(\xi) \tau_{2}+N_{2}(\xi) \tau_{8}+\ldots+N_{8}(\xi) \tau_{44} \\
& \ldots \\
& \tau_{13}(x)=N_{1}(\xi) \tau_{6}+N_{2}(\xi) \tau_{12}+\ldots+N_{8}(\xi) \tau_{48} \\
& \tau_{21}=\tau_{12}, \tau_{31}=\tau_{13}, \tau_{32}=\tau_{23}
\end{aligned}
$$

within an $e$-th element $\Omega_{e} \subset \Omega \subset \mathbb{R}^{3}$, where $\xi=$ $\left(\xi_{1}, \xi_{2}, \xi_{3}\right) \in \omega$ parameterize the master element $\omega=$ $[-1,1] \times[-1,1] \times[-1,1], x=\left(x_{1}, x_{2}, x_{3}\right)=x^{e}(\xi) \in \Omega_{e}$ parameterize real elements $\Omega_{e}$ being the images of the master element $\omega$ under the geometric mappings $x^{e}: \omega \rightarrow \Omega_{e}$ and $\tau_{6 i-5}, \tau_{6 i-4}, \tau_{6 i-3}, \tau_{6 i-2}, \tau_{6 i-1}, \tau_{6 i}$ are the unknown nodal stresses $\tau_{11}, \tau_{22}, \tau_{33}, \tau_{23}, \tau_{12}, \tau_{13}$ at nodes $1,2, \ldots, 8$, respectively (we simplify notation and omit upper index writing e.g. $\tau_{j}$ instead of writing more correctly $\tau_{j}^{e}$ ). The virtual displacement field $\boldsymbol{v}=\left(v_{1}, v_{2}, v_{3}\right)$ in variational equation (II.5) is interpolated within an $e$-th element $\Omega_{e}$ similarly as the stress field

$$
\begin{aligned}
& v_{1}(x)=N_{1}(\xi) w_{1}+N_{2}(\xi) w_{4}+\ldots+N_{8}(\xi) w_{22} \\
& v_{2}(x)=N_{1}(\xi) w_{2}+N_{2}(\xi) w_{5}+\ldots+N_{8}(\xi) w_{23} \\
& v_{3}(x)=N_{1}(\xi) w_{3}+N_{2}(\xi) w_{6}+\ldots+N_{8}(\xi) w_{24}
\end{aligned}
$$

where $w_{3 i-2}, w_{3 i-1}, w_{3 i}$ are the unknown nodal virtual displacements $v_{1}, v_{2}, v_{3}$ at the nodes $1,2,3$, respectively. The components of the derivative $(\delta \varepsilon)_{i j}$ are computed by the following formulae

$$
\begin{aligned}
& {\left[\begin{array}{c}
(\delta \varepsilon)_{i 1}(x) \\
(\delta \varepsilon)_{i 2}(x) \\
(\delta \varepsilon)_{i 3}(x)
\end{array}\right]=\left[\begin{array}{lll}
j_{11} & j_{12} & j_{13} \\
j_{21} & j_{22} & j_{23} \\
j_{31} & j_{32} & j_{33}
\end{array}\right]\left[\begin{array}{l}
v_{i, \xi_{1}} \\
v_{i, \xi_{2}} \\
v_{i, \xi_{3}}
\end{array}\right]} \\
& (i=1,2,3)
\end{aligned}
$$

where $j_{k l}=j_{k l}(\xi)$ are the components of the $3 \times 3$ matrices $\boldsymbol{j}^{e}=\boldsymbol{j}^{e}(\xi)$ being the inverse and transposed Jacobian matrices defined by the partial derivatives $\frac{\partial x_{i}^{e}}{\partial \xi_{j}}(i, j=1,2,3)$ of the transformation $x^{e}=\left(x_{1}^{e}, x_{2}^{e}, x_{3}^{e}\right)$ defining the shape of the body $\Omega$ (we have omitted the upper index $e$ in $j_{k l}^{e}$ ). Substitution of (III.1)-(III.4) into the variational equation (II.5) results in the set of linear equations representing the equilibrium conditions

$$
B t=Q
$$

where $\boldsymbol{B} \in \mathbb{R}^{M \times n}$ is the rectangular $M \times n$ statics matrix, $\boldsymbol{Q} \in \mathbb{R}^{M}, \boldsymbol{t} \in \mathbb{R}^{n}$ are vectors of nodal forces (only partially known) and unknown nodal parameters $\tau_{j}$ defining the stress fields, respectively. Number $M$ of rows and number $n$ of columns (unknown nodal parameters $\tau_{j}, j=1, \ldots, n$ in global notation) in the matrix $\boldsymbol{B}$ are equal $3 \times N$ and $6 \times N$, respectively, where $N$ denotes the number of all nodes in the global finite element mesh. Similarly as in the Simplex
Method we perform the partition of the rectangular matrix $B$ and vector $\boldsymbol{Q}$ into two matrices: upper $\boldsymbol{B}^{u} \in \mathbb{R}^{m \times n}$, lower $\boldsymbol{B}^{l} \in \mathbb{R}^{(M-m) \times n}$ and two vectors, upper $\boldsymbol{Q}^{u} \in \mathbb{R}^{m}$ and lower $\boldsymbol{Q}^{l} \in \mathbb{R}^{(M-m)}$, respectively. The first $m$ indices of the rows in upper matrix $\boldsymbol{B}^{u}$ correspond to the indices defining the global, unknown, free degrees of freedom and the remaining $M-m$ indices of the rows in lower matrix $\boldsymbol{B}^{l}$ correspond to the indices of the global, known, constrained degrees of freedom (boundary conditions). All components of the vector $\boldsymbol{Q}^{u}$ are known and the vector of the unknown boundary reactions can be calculated from the relation $\boldsymbol{Q}^{l}=\boldsymbol{B}^{l} \boldsymbol{t}$ upon finding the vector $t$ from the system of rectangular linear equations $\boldsymbol{B}^{u} \boldsymbol{t}=\boldsymbol{Q}^{u}$. In the Force Method, the number $M-m$ defines the degree of statical indeterminacy of the bar structure and could obviously be equal to 0 . In the considered problem the number $M-m$ is always positive. The set of all solutions of the equations

$$
\boldsymbol{B}^{u} \boldsymbol{t}=\boldsymbol{Q}^{u}
$$

can be expressed as

$$
\Theta=\left\{\boldsymbol{t}=\boldsymbol{t}\left(\alpha_{1}, \ldots, \alpha_{s}\right) \mid \boldsymbol{t}=\boldsymbol{t}^{\circ}+\sum_{k=1}^{s} \alpha_{k} \boldsymbol{h}_{k}, \alpha_{k} \in \mathbb{R}\right\},
$$

where $\boldsymbol{h}_{k}=\left[\begin{array}{llll}h_{1 k} & h_{2 k} & \ldots & h_{n k}\end{array}\right]^{T} \in \mathbb{R}^{n}, \quad k=$ $1,2, \ldots, s$ are the vectors that span the $s$-dimensional kernel of the matrix $\boldsymbol{B}^{u}$ and $\boldsymbol{t}^{\circ}=\left[\begin{array}{llll}t_{1}^{\circ} & t_{2}^{\circ} & \ldots & t_{n}^{\circ}\end{array}\right]^{T} \in \mathbb{R}^{n}$ is the arbitrary, fundamental solution of the set of linear equations $\boldsymbol{B}^{u} \boldsymbol{t}=\boldsymbol{Q}^{u}$. In each $e$-th finite element $\Omega_{e}$, the stress components (III.2) depend not only on $\xi \in \omega$ and local nodal parameters $\tau_{j}^{e}(j=1, \ldots, 48)$ but additionally on global parameters $\alpha_{k} \quad(k=1, \ldots, s)$ defining the linear combinations of the particular local components $h_{j k}(j=1, \ldots, n, \quad k=1, \ldots, s)$. In other words, upon constructing the solution (found only once) of linear, rectangular algebraic system $\boldsymbol{B}^{u} \boldsymbol{t}=\boldsymbol{Q}^{u}$, one obtains a very simple approximation $\Sigma^{\alpha}(\Omega)$ of the statically admissible set of the stress fields $\Sigma(\Omega)$ determined by $s$ global parameters $\alpha_{k} \in \mathbb{R}$

$$
\Sigma^{\boldsymbol{\alpha}}(\Omega)=\left\{\boldsymbol{\tau}=\boldsymbol{\tau}(\boldsymbol{\alpha}) \in S^{3} \mid \boldsymbol{\alpha}=\left(\alpha_{1}, \ldots, \alpha_{s}\right) \in \mathbb{R}^{s}\right\}
$$

where

$$
\begin{aligned}
\tau_{11} & =N_{1} t_{1}^{\circ}+\ldots+N_{8} t_{43}^{\circ}+\sum_{k=1}^{s} \alpha_{k} N_{1} h_{I_{1} k}+\ldots \\
& +\sum_{k=1}^{s} \alpha_{k} N_{8} h_{I_{43} k} \\
\tau_{22}= & N_{1} t_{2}^{\circ}+\ldots+N_{8} t_{44}^{\circ}+\sum_{k=1}^{s} \alpha_{k} N_{1} h_{I_{2} k}+\ldots \\
& +\sum_{k=1}^{s} \alpha_{k} N_{8} h_{I_{44} k} \\
& \quad \ldots
\end{aligned}
$$




$$
\begin{aligned}
\tau_{13}= & N_{1} t_{6}^{\circ}+\ldots+N_{8} t_{48}^{\circ}+\sum_{k=1}^{s} \alpha_{k} N_{1} h_{I_{6} k}+\ldots \\
& +\sum_{k=1}^{s} \alpha_{k} N_{8} h_{I_{48} k} \\
& \tau_{21}=\tau_{12}, \tau_{31}=\tau_{13}, \tau_{32}=\tau_{23} .
\end{aligned}
$$

Note that $j$ in $t_{j}^{\circ}$ runs over $\{1,2, \ldots, 48\}$, while $j$ in $h_{j k}$ runs over $\{1,2, \ldots, n\}$.

The constrained variational problem (II.31a) is approximated by the family of discretized problems which assume the form of the unconstrained algebraic problems

$$
S_{\boldsymbol{\alpha}}^{*}=\min _{\boldsymbol{\tau} \in \Sigma^{\boldsymbol{\alpha}}(\Omega)} \int_{\Omega} \Pi(\boldsymbol{\tau}) \mathrm{d} x
$$

They can be equivalently written as (see (III.7)): find $\boldsymbol{\alpha}^{*} \in \mathbb{R}^{s}$ such that

$$
S_{\boldsymbol{\alpha}^{*}}^{*}=\min _{\boldsymbol{\alpha} \in \mathbb{R}^{s}} \int_{\Omega} \Pi^{\tau}(\boldsymbol{\alpha}) \mathrm{d} x
$$

where the integrand is defined as follows

$$
\Pi^{\tau}: \mathbb{R}^{s} \rightarrow \mathbb{R} \quad, \quad \Pi^{\tau}=\Pi \circ \tau
$$

(we have omitted in the notation the obvious dependence on $x \in \Omega$ in $\Pi(x, \boldsymbol{\tau})$ and $\left.\Pi^{\boldsymbol{\tau}}(x, \boldsymbol{\alpha})\right)$. Integration in (III.9) is performed numerically, i.e.

$$
\int_{\Omega} \Pi^{\boldsymbol{\tau}}(\boldsymbol{\alpha}) \mathrm{d} x \cong \sum_{e} \sum_{\zeta \in \omega} w(\zeta) \Pi^{\boldsymbol{\tau}}(\zeta, \boldsymbol{\alpha})\left|\operatorname{det} \boldsymbol{j}^{e}(\zeta)\right|
$$

where $\zeta=\left(\zeta_{1}, \zeta_{2}, \zeta_{3}\right) \in \omega$ and $w=w(\zeta)$ are Gauss integration points and weights, respectively. At each arbitrary, but fixed point $\zeta \in \omega$, the gradient

$$
\begin{aligned}
& \operatorname{grad} \Pi^{\boldsymbol{\tau}}=\operatorname{grad} \Pi^{\boldsymbol{\tau}}(\zeta, \boldsymbol{\alpha})= \\
= & {\left[\begin{array}{llll}
\frac{\partial \Pi^{\boldsymbol{\tau}}}{\partial \alpha_{1}}(\zeta, \boldsymbol{\alpha}) & \frac{\partial \Pi^{\boldsymbol{\tau}}}{\partial \alpha_{2}}(\zeta, \boldsymbol{\alpha}) & \ldots & \frac{\partial \Pi^{\boldsymbol{\tau}}}{\partial \alpha_{s}}(\zeta, \boldsymbol{\alpha})
\end{array}\right]^{T} \in \mathbb{R}^{s} }
\end{aligned}
$$

of the functional (III.10) can be easily computed. The formula is the following (as in (III.9) we omit the dependence on $\zeta \in \omega)$ :

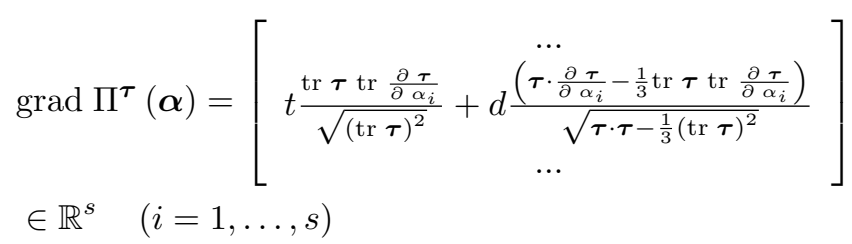

$(t=\sqrt{10}, d=5 \sqrt{6})$, where (see (III.8))

$$
\begin{aligned}
& \frac{\partial \tau_{11}}{\partial \alpha_{k}}=N_{1} h_{I_{1} k}+N_{2} h_{I_{7} k}+\ldots+N_{8} h_{I_{43} k} \\
& \frac{\partial \tau_{22}}{\partial \alpha_{k}}=N_{1} h_{I_{2} k}+N_{2} h_{I_{8} k}+\ldots+N_{8} h_{I_{44} k} \\
& \ldots \\
& \frac{\partial \tau_{13}}{\partial \alpha_{k}}=N_{1} h_{I_{6} k}+N_{2} h_{I_{12} k}+\ldots+N_{8} h_{I_{48} k} .
\end{aligned}
$$

The formulae (III.11) and (III.13)-(III.14) make it possible to calculate the values of the function

$$
\Xi: \mathbb{R}^{s} \rightarrow \mathbb{R}, \Xi(\boldsymbol{\alpha})=\sum_{e} \sum_{\zeta \in \omega} w(\zeta) \Pi^{\boldsymbol{\tau}}(\zeta, \boldsymbol{\alpha})\left|\operatorname{det} \boldsymbol{j}^{e}(\zeta)\right|
$$

and $s$ components of its gradient

$\operatorname{grad} \Xi(\boldsymbol{\alpha})=\sum_{e} \sum_{\zeta \in \omega} w(\zeta) \operatorname{grad} \Pi^{\boldsymbol{\tau}}(\zeta, \boldsymbol{\alpha})\left|\operatorname{det} \boldsymbol{j}^{e}(\zeta)\right| \in \mathbb{R}^{s}$

in problem (III.9) re-written now as: find $\boldsymbol{\alpha}^{*} \in \mathbb{R}^{s}$ such that

$$
S_{\boldsymbol{\alpha}^{*}}^{*}=\min _{\boldsymbol{\alpha} \in \mathbb{R}^{s}} \Xi(\boldsymbol{\alpha})
$$

\section{THE ISOTROPIC MATERIAL DESIGN ALGORITHM (IMD ALGORITHM)}

The computational procedure implementing the IMD algorithm consists of the following main steps

Step 1. Set the rectangular system of linear algebraic equations (III.5) or $\boldsymbol{B} \boldsymbol{t}=\boldsymbol{Q}$, in accordance with FEM.

Step 2. Separate upper sub-matrix $\boldsymbol{B}^{u}$ and upper sub-vector $Q^{u}$ corresponding to the unknown degrees of freedom of the FEM.

Step 3. Find the solutions $\boldsymbol{t}=\boldsymbol{t}^{\circ}+\sum_{k=1}^{s} \alpha_{k} \boldsymbol{h}_{k}$ of the rectangular system of linear algebraic equations $B^{u} t=Q^{u}$. Step 4. Apply any algorithm of the nonlinear mathematical programming to find the solution $\boldsymbol{\alpha}^{*} \in \mathbb{R}^{s}$ of the minimization problem $S_{\boldsymbol{\alpha}^{*}}^{*}=\min _{\boldsymbol{\alpha} \in \mathbb{R}^{s}} \Xi(\boldsymbol{\alpha})$, where the function $\Xi(\boldsymbol{\alpha})$ and its gradient grad $\Xi(\boldsymbol{\alpha})$ are defined by (III.15), (III.16), respectively.

Step 5. In accordance with the formulae (III.8), find all components

$$
\begin{aligned}
& \tau_{11}^{*}=\tau_{11}\left(\boldsymbol{\alpha}^{*}\right), \tau_{22}^{*}=\tau_{22}\left(\boldsymbol{\alpha}^{*}\right), \\
& \tau_{33}^{*}=\tau_{33}\left(\boldsymbol{\alpha}^{*}\right), \tau_{23}^{*}=\tau_{23}\left(\boldsymbol{\alpha}^{*}\right), \\
& \tau_{12}^{*}=\tau_{12}\left(\boldsymbol{\alpha}^{*}\right), \tau_{13}^{*}=\tau_{13}\left(\boldsymbol{\alpha}^{*}\right), \\
& \tau_{21}^{*}=\tau_{12}^{*}, \tau_{31}^{*}=\tau_{13}^{*}, \tau_{32}^{*}=\tau_{23}^{*}
\end{aligned}
$$

defined by the optimal solution $\boldsymbol{\alpha}^{*} \in \mathbb{R}^{s}$ found in step 4 .

Step 6. In accordance with the formulae (II.29) find the distribution of the optimal bulk and shear moduli $k^{*}=$ $k_{\boldsymbol{\tau}}^{*}\left(\boldsymbol{\tau}^{*}\right), \mu^{*}=\mu_{\boldsymbol{\tau}}^{*}\left(\boldsymbol{\tau}^{*}\right)$, respectively.

Step 7. In accordance with the formula (II.33) find the distribution of the components of the optimal Hooke tensor $\boldsymbol{H}^{*}$.

For the optimal, non-homogeneous, anisotropic Hooke tensor $C^{*}$ (see (II.39)), it is possible to find the closest nonhomogeneous, isotropic Hooke tensor $\boldsymbol{D}$ characterized by bulk 
and shear moduli $k, \mu$ or Young modulus $E$ and Poisson ratio $\nu$. "Closest" means that the shear moduli $k, \mu$ defining the isotropic matrix

$$
\begin{aligned}
\widehat{\boldsymbol{D}} & =\left[\begin{array}{cccccc}
k+\frac{4}{3} \mu & k-\frac{2}{3} \mu & k-\frac{2}{3} \mu & 0 & 0 & 0 \\
k-\frac{2}{3} \mu & k+\frac{4}{3} \mu & k-\frac{2}{3} \mu & 0 & 0 & 0 \\
k-\frac{2}{3} \mu & k-\frac{2}{3} \mu & k+\frac{4}{3} \mu & 0 & 0 & 0 \\
0 & 0 & 0 & 2 \mu & 0 & 0 \\
0 & 0 & 0 & 0 & 2 \mu & 0 \\
0 & 0 & 0 & 0 & 0 & 2 \mu
\end{array}\right] \\
& \in \mathbb{R}^{6 \times 6}
\end{aligned}
$$
are minimizers found from the condition $\min _{k, \mu}\left\|\widehat{C^{*}}-\widehat{D}\right\|$, expressed by the Frobenius norm; matrix $\widehat{C}^{*}$ has the same components as in (II.4) after replacing $C_{i j k l}$ by $C_{i j k l}^{*}$. It is not difficult to show that the minimizers $k, \mu$ of this problem read (see [11]):

$$
\begin{aligned}
k & =\frac{1}{9}\left(C_{1111}^{*}+C_{2222}^{*}+C_{3333}^{*}\right. \\
& \left.+2\left(C_{2233}^{*}+C_{1122}^{*}+C_{1133}^{*}\right)\right), \\
\mu & =\frac{1}{15}\left(C_{1111}^{*}+C_{2222}^{*}+C_{3333}^{*}\right. \\
& \left.-\left(C_{2233}^{*}+C_{1122}^{*}+C_{1133}^{*}\right)\right) \\
& +\frac{1}{5}\left(C_{2323}^{*}+C_{1313}^{*}+C_{1212}^{*}\right) .
\end{aligned}
$$

It should be noted that in this case the value of the trace of the elastic moduli tensor is preserved at each point of the $\Omega$ domain, i.e.

$$
\forall x \in \Omega \quad \operatorname{tr} \boldsymbol{D}(x)=3 k(x)+10 \mu(x)=\operatorname{tr} \boldsymbol{C}^{*}(x),
$$

which can be immediately verified after simple calculations. Young modulus $E$ and Poisson ratio $\nu$ for such closest, nonhomogeneous, isotropic material could then be easily calculated by (II.17).

Now, we define the non-optimal, homogeneous, isotropic Hooke tensor $\boldsymbol{H}$, satisfying only the isoperimetric condition

$$
\Re(\boldsymbol{H})=\int_{\Omega} \operatorname{tr} \boldsymbol{H} \mathrm{d} x-\Lambda=0
$$

to compare the optimal and non-optimal compliances of the body $\Omega$. For this purpose, let us assume that the Poisson ratio $\nu=\bar{\nu}$ is known and constant in $\Omega$. We also assume that the fields $k=\bar{k}$ and $\mu=\bar{\mu}$ (and thus the field $E=\bar{E}$ ) defining the tensor $\boldsymbol{H}$ are constant in $\Omega$. For a given elastic modulus $E_{0}$ we can always calculate $\Lambda=E_{0}|\Omega|$ (see (II.9) 2 ). Thus, on the basis of condition (IV.5) and (II.21) we conclude that

$$
\int_{\Omega}(3 \bar{k}+10 \bar{\mu}) \mathrm{d} x=(3 \bar{k}+10 \bar{\mu})|\Omega|=E_{0}|\Omega|=\Lambda
$$

and after replacing $\bar{k}$ and $\bar{\mu}$ by $\bar{E} / 3(1-2 \bar{\nu})$ and $\bar{E} / 2(1+\bar{\nu})$, respectively (see (II.17) 1,2 ), we can easily calculate (constant in $\Omega$ ) Young modulus $\bar{E}=\bar{E}\left(E_{0}, \bar{\nu}\right)$ from the relation (IV.6) as

$$
\bar{E}=\frac{(2 \bar{\nu}-1)(\bar{\nu}+1)}{3(3 \bar{\nu}-2)} E_{0} .
$$

The property (IV.4) together with the formulae (IV.3), (II.17) and condition (IV.5) together with the formula (IV.7) for known constant Poisson ratio $\bar{\nu}$ allows to compare the four compliances:

1. $\wp^{*}=\wp_{C}^{*}$ for the optimal, non-homogeneous, anisotropic tensor $C^{*}$

2. $\wp^{*}=\wp_{H^{*}}$ for the optimal, non-homogeneous, isotropic tensor $\boldsymbol{H}^{*}$

3. $\wp_{D}$ for the non-homogeneous, isotropic tensor $\boldsymbol{D}$ closest in the sense of Frobenius metric to the optimal, non-homogeneous, anisotropic tensor $C^{*}$

4. $\wp_{\boldsymbol{H}}$ for the homogeneous, isotropic tensor $\boldsymbol{H}$ while fulfilling all four isoperimetric conditions for all of the above cases, i.e.

$$
\Re\left(\boldsymbol{C}^{*}\right)=\Re\left(\boldsymbol{H}^{*}\right)=\Re(\boldsymbol{D})=\Re(\boldsymbol{H})=0 .
$$

\section{CASE STUDY}

The aim of this section is to show the optimal layouts of the optimal moduli $\lambda_{1}^{*}, k^{*}, \mu^{*}, E^{*}, \nu^{*}$ within short cubic cantilever (supported on bottom side) of length $L_{x}=1.0[\mathrm{~m}]$, width $L_{y}=1.0[\mathrm{~m}]$ and height $L_{z}=1.0[\mathrm{~m}]$ (see Fig. 1). The finite element mesh is defined by $10 \times 10 \times 10=1000$ cubic modules. The horizontal, constant and uniformly applied loading $T_{1}=1.0\left[\mathrm{~N} / \mathrm{m}^{2}\right]$ on upper side is applied. The 8- and 4-points rules of the Gauss integration for the three-dimensional cube and two-dimensional upper square are adopted, respectively. The unity value of elastic modulus $E_{0}$ in (II.9) $)_{2}$ was assumed, i.e. $E_{0}=1.0\left[\mathrm{~N} / \mathrm{m}^{2}\right]$. The optimal values of the design moduli and optimal compliances are proportional to $E_{0}$ or to its inverse (see (II.40), (II.29), (II.42) and (II.30)) so to get the results for the practical materials, it is sufficient to multiply the obtained numerical values by $E_{0}>>1.0$ or its inverse. The non-optimal, homogeneous, isotropic tensor $\boldsymbol{H}$ is defined by (constant in $\Omega$ ) Young modulus $\bar{E}=\bar{E}\left(E_{0}, \bar{\nu}\right)$ calculated from the formula (IV.7) for (constant in $\Omega$ ) Poisson ratio $\bar{\nu}=0.3$.

The number of the main iteration loops for the assumed tolerance $1.0 \cdot 10^{-5}$ was equal 32 and 42 for anisotropic and isotropic body, respectively. Layouts have been found with using the graphical program: Voxler Ver 1.1.1716, Golden Software, Inc.

The optimal compliances are: $\wp_{\boldsymbol{C}^{*}}=4.87161, \wp_{\boldsymbol{H}^{*}}=$ $27.5471, \wp_{\boldsymbol{D}}=27.1577$ and $\wp_{\boldsymbol{H}}=42.7515$ [Nm] for optimal anisotropic, optimal isotropic, isotropic closest to optimal 

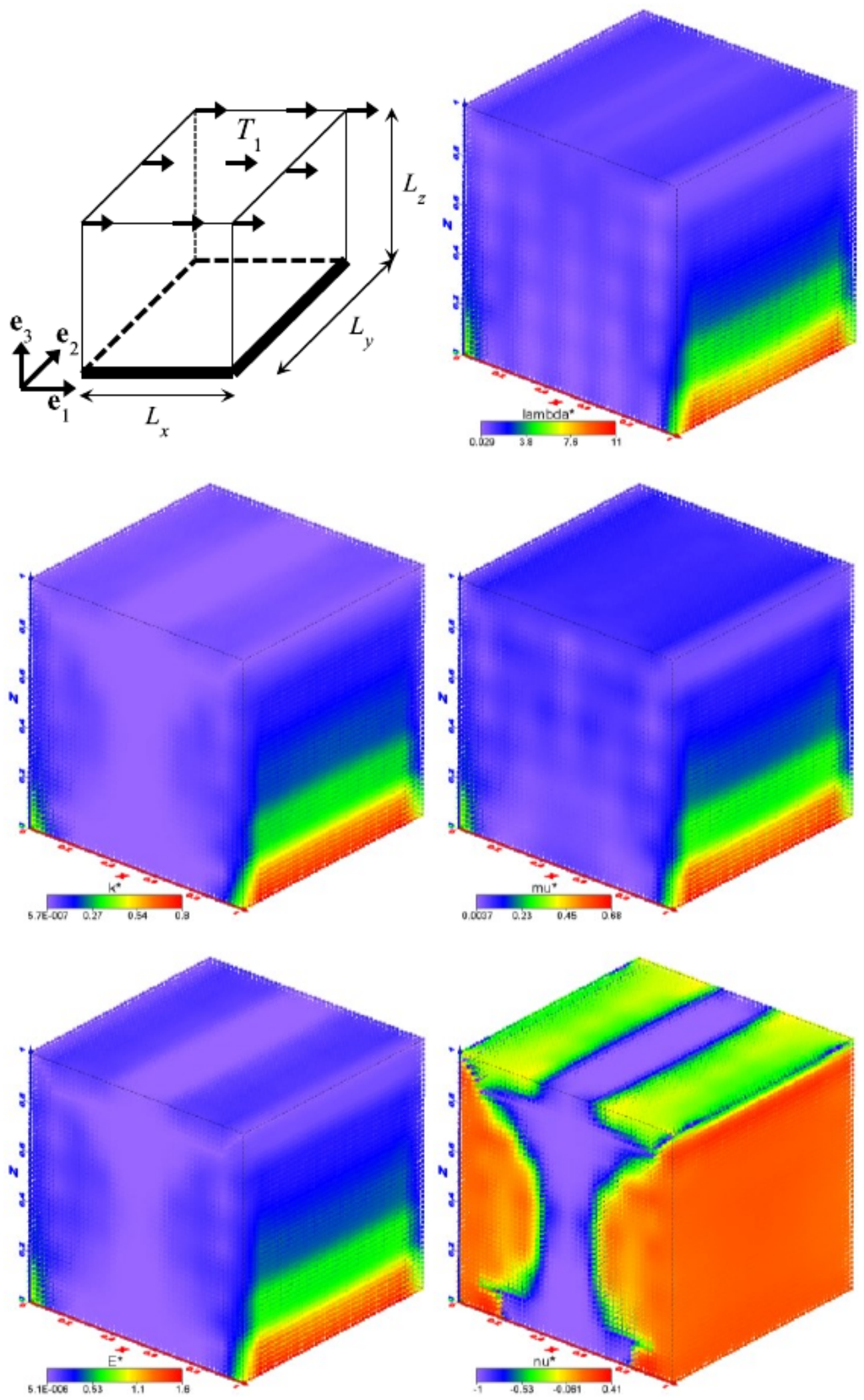

Fig. 1. The body $\Omega$ - cube $L_{x} \times L_{y} \times L_{z}=1 \times 1 \times 1[\mathrm{~m}]$ with uniformly applied constant loading $T_{1}=1\left[N / \mathrm{m}^{2}\right]$ (left upper figure). In remaining figures: the scatter plots of the optimal Kelvin modulus $\lambda_{1}^{*}$ (by FMD method), optimal bulk and shear moduli $k^{*}, \mu^{*}$, optimal Young modulus $E^{*}$ and optimal Poisson ration $\nu^{*}$ (by IMD method), respectively 
anisotropic and non-optimal, homogeneous isotropic body, respectively. It occurs that the optimal Poisson ratio $\nu^{*}$ assumes the values from the whole admissible range: $(-1,1 / 2)$ in a large part of the design domain $\Omega$. Thus the IMD method confirms the importance of the auxetic materials, see e.g. [13]. For more examples of optimal distributions of elastic moduli $k^{*}, \mu^{*}$ and $E^{*}, \nu^{*}$ revealing the auxetic characteristic of the stiffest bodies, see the paper [14].

The optimal layouts $k^{*}$ and $\mu^{*}$ or $E^{*}$ and $\nu^{*}$ are characterized by extremely abrupt changes of their values on very small subdomains in $\Omega$. Furthermore, its values for fairly large subdomains reach zero value. These features allow to classify these optimal materials as Functionally Graded Materials (FGM) characterized by the variation in composition and structure over volume, resulting in corresponding changes in its properties - see e.g. [15] where an interesting technique based on the assignment of material attributes to the quantized volume was presented making the IMD designs FGM-manufacturable.

The numerical approach of step 3 is based upon two well-known theorems of linear algebra for any $m \times n$ matrix $\boldsymbol{B}$, where $m \leq n$. Let us assume that the dimension $s$ of the kernel of the matrix $\boldsymbol{B}$ is equal to $n-m$, i.e. $s=\operatorname{dim} \operatorname{ker} B=n-m$. Then

Theorem I (SVD decompositions). Any matrix $\boldsymbol{B} \in \mathbb{R}^{m \times n}$ can be written as the product $\boldsymbol{B}=\boldsymbol{U} \boldsymbol{W} \boldsymbol{V}^{T}$ of an $m \times n$ orthogonal matrix $\boldsymbol{U} \in \mathbb{R}^{m \times n}$, an $n \times n$ diagonal matrix $\boldsymbol{W} \in \mathbb{R}^{n \times n}$ with positive or zero elements and the transpose of an $n \times n$ orthogonal matrix $\boldsymbol{V} \in \mathbb{R}^{n \times n}$.

Theorem II (QR decompositions). Transposition $\boldsymbol{B}^{T} \in$ $\mathbb{R}^{n \times m}$ of any matrix $\boldsymbol{B} \in \mathbb{R}^{m \times n}$ can be written as the product $\boldsymbol{B}^{T}=\boldsymbol{Q} \boldsymbol{R}$ of an unitary $n \times n$ matrix $\boldsymbol{Q}=\left[\begin{array}{ll}\boldsymbol{Q}_{1} & \boldsymbol{Q}_{2}\end{array}\right] \in \mathbb{R}^{n \times n}$, with both matrices $\boldsymbol{Q}_{1} \in \mathbb{R}^{n \times m}$, $\boldsymbol{Q}_{2} \in \mathbb{R}^{n \times(n-m)}$ having the orthogonal columns and upper triangular $n \times m$ matrix $\boldsymbol{R}=\left[\begin{array}{c}\boldsymbol{R}_{1} \\ \mathbf{0}\end{array}\right] \in \mathbb{R}^{n \times m}$, where $\forall i=1, \ldots, m \quad R_{i i}>0$.

It can be shown that $s$ columns $\boldsymbol{V}_{j} \in \mathbb{R}^{n}\left(j=j_{k}\right.$, $k=1, \ldots, s)$ of the matrix $\boldsymbol{V}$ that correspond to $W_{j_{k} j_{k}}=0$ and $s$ columns $\boldsymbol{Q}_{2}^{1}, \boldsymbol{Q}_{2}^{2}, \ldots, \boldsymbol{Q}_{2}^{s} \in \mathbb{R}^{n}$ of the matrix $\boldsymbol{Q}_{2}$ span the kernel ker $\boldsymbol{B}$ of the matrix $\boldsymbol{B}$ in SVD and QR decomposition, respectively. This fact enables to find numerically the set of all solutions of the equation (III.6) taking matrix $\boldsymbol{B}$ above as $\boldsymbol{B}^{u}$ in (III.6). The routine svdcmp(...) in C language from [16], p. 67 in the case of SVD algorithm was implemented. In finding the numerical solution in step 4, three various, gradient-oriented numerical routines in $\mathrm{C}++$ language: frprmn(...) or dfpmin(...) (see [16], p. 423 and 428) and own routine implementing the steepest descent algorithm $\boldsymbol{\alpha}_{k+1}=\boldsymbol{\alpha}_{k}-\rho_{k} \operatorname{grad} \Xi\left(\boldsymbol{\alpha}_{k}\right)$ with the simple learning rule determining the learning rate $\rho_{k}$ for obtaining the opti- mal solution $\boldsymbol{\alpha}^{*}=\left(\alpha_{1}^{*}, \alpha_{2}^{*}, \ldots, \alpha_{s}^{*}\right) \in \mathbb{R}^{s}$ were implemented. The first two routines implement the Fletcher-Reeves (FR), Polak-Ribiere (PR) or Broyden-Fletcher-Goldfarb-Shanno (BFGS) algorithms of non-linear mathematical programming. The routines frprmn(...) or dfpmin(...) was always called for the zero initial values of $\alpha_{i}, i=1, \ldots, s$ (randomly generated values of $\alpha_{i}$ gave almost the same numerical solution).

\section{FINAL REMARKS}

The stress-based FMD proposed in $[9,10]$ and its isotropic version IMD put forward in the present paper differ considerably from the displacement-based FMD, cf. [1, 5, 7], due to the following features:

a) The original optimization problem (II.10) with an isoperimetric conditions is reduced to the auxiliary optimization problem (II.31b) free of such a condition.

b) The auxiliary problem (II.31b) is replaced by the discretized problem (III.17) free of any bounds on the design variables, including box-like constraints.

c) A clear decomposition of the algorithm into two independent problems concerning statics and optimization.

In the first problem the statically admissible approximation of stresses, determined by the coefficients of the linear combinations of vectors of the kernel of the equilibrium matrix of the body, is constructed, which constitutes the steps 1-3. It should be stressed that this problem is solved once, in contrast to the displacement based FMD algorithms in which the equilibrium problem must be solved in each main loop of the algorithm.

In the optimization problem the coefficients at the basis vectors of the kernel of matrix $\boldsymbol{B}^{u}$ are found by the condition of the minimum of the function (III.15), i.e. step 4. Since the formulae for the gradient of this function are available, one can implement arbitrary gradient-based algorithm aimed at solving the unconstrained minimization problems.

d) Both the displacement and strain fields are eliminated from the algorithm. The only fields involved at each step of the FMD and IMD algorithms are the trial stress fields. The stress approximant minimizing the auxiliary function (III.15) (see also (II.31), (II.32)) determines all the unknown optimal material characteristics at each point of the design domain, which is done in steps 5-7

e) Necessity of implementing numerical algorithms for solving algebraic under-determinate systems of algebraic equations. Moreover, in 3D problems the number of stress unknowns is essentially bigger than the number of unknowns involved in any displacement-based method (step 3). For instance, approximation of the trial stress fields in 3D (Eqs. (III.2)) necessitates at least six nodal parameters, while in the conventional FEM three nodal parameters suffice for displacements approximation (see Eq. (III.3)). Let us emphasize here that there are no available, efficient and numerically 
stable procedures of constructing the kernel of the matrix of an under-determinate algebraic system. While comparing with the extremely rich and easy available libraries (like LAPACK and PETSc) of numerical procedures to solve the Cramer's systems (i.e. the algebraic systems with square matrices) we note the lack of similar programs for the underdeterminate systems, which is the main obstacle in increasing the FEM mesh density in the stress-based FMD. This may be, however, alleviated by the basic property of the stress-based approach: to find stresses we do not need differentiation of the displacement fields. Yet this needs an additional discussion, which lies beyond the scope of the present paper.

The present paper has not dealt with the problem of convergence of the family of approximate solutions $t \in \mathbb{R}^{n}$ approximating the stress fields, being parameterized by, depending on the mesh density, dimension of the kernel of the matrix $\boldsymbol{B}^{u} \in \mathbb{R}^{m \times n}$. The hitherto performed tests published in [8] concerned the 2D FMD problems (in the anisotropic setting); the process of making the uniform meshes of the conforming C2D4 element denser and denser contributed to a visible stabilization of the isolines of distribution of the optimal values of the Kelvin modulus $\lambda_{1}^{*}$ and of the target functional (II.41). In the 3D case such a tendency has also been noted, yet similarly dense meshes could not be applied due to limited equipment parameters (Laptop Intel(R) Core(TM)2 Duo CPU T9400@2.53GHz). Dimensions of the rectangular matrix $\boldsymbol{B}^{u}$ used in the examples presented above were close to the maximal dimensions admissible by the 4 GB RAM. The run time was conditioned by the computational complexity of order $\mathcal{O}\left(m^{2} \times n+n^{3}\right)$, implemented in the SVD algorithm. To overcome the mentioned difficulties the developed C++ program is being adjusted to the supercomputers available at the Interdisciplinary Centre for Mathematical and Computational Modelling of the University of Warsaw (ICM); the program is based on the $\mathrm{QR}$ decomposition, available within the LAPACK library having a much smaller computational complexity of order $\mathcal{O}\left(2 \times m \times n^{2}\right)$ than the SVD method.

The first tests have confirmed that much denser meshes can be used, but along with the increase of the number of unknowns new problems linked with the computational complexity of the optimizers arise. The computation time used to solve the QR problems has now become much smaller than the time necessary to perform the optimization algorithms.

The performed numerical tests have shown some important features of the optimal solutions and make it possible to compare the optimal anisotropic designs (solutions to the FMD problem) with the optimal isotropic ones (or solutions to the IMD problems). It turns out that the FMD designs are 5 or even 6 times stiffer than their IMD counterparts, the latter being about 2 times stiffer than the homogeneous isotropic designs (case of $\bar{\nu}=0.3$ ). On the other hand, however, the anisotropic FM designs are appropriate only for the given load, since the Hooke tensor becomes degenerated; only one of Kelvin moduli $\lambda_{K}$ is positive. This degeneracy is com- patible with the loading, hence does not theoretically hinder from finding the stress fields and selected components of the displacement field. Nevertheless, appropriate tools are necessary to deal with the conditionally stable numerical scheme of the FMD method; the global stiffness matrix of FEM becomes singular or numerically very close to singular, see Press et al [16]. To overcome the difficulties we have to replace the conventional LU decomposition by the numerically stable SVD method for the square stiffness matrix of the FEM to achieve reliable values of nodal displacements. We have to reckon with non-uniqueness of the solution. It has the properties known from the analysis of geometrically variable trusses. The implementation of the LU decomposition (like ludcmp(...) in [16]) results in the uncontrolled, random values of selected components of the displacement vector; these components are directed orthogonally to the trajectories of the stress field (coinciding with optimal anisotropy lines). The displacements measured along the non-zero stress trajectories could be computed with sufficient accuracy. Having them, one could compute the compliance by (II.6) and, in all cases, this value turned out to be almost equal to the value of the compliance computed by (II.42), which was found as a result of solving the stress based auxiliary problem. The above problems are not present in the IMD method. This method does not lead to any degeneracy of the stiffness matrix. Also within this approach the compliance has been computed by two manners: having the displacements-then by (II.6), and having solved the stress based problem- by (II.30). It is worth noting that the discussed numerical mismatch noted in the IMD is in all cases bigger than in the FMD method.

The results of the IMD have been confronted with the isotropic design (see (IV.2), (IV.4)) constructed as the closest, in the sense of the distance measured by the Frobenius norm, to the anisotropic FM design. The compliance of the isotropic non-homogeneous structure thus found could be computed by solving the FEM problem. It turns out that this value is in all cases very close to the IMD result and always slightly bigger. Moreover, the layouts of the optimal moduli $E^{*}$ and $E$ as well as the Poisson ratios $\nu^{*}$ and $\nu$ for the corresponding methods are almost identical, which suggests they could be identical. This conjecture has not been proved yet.

Closing the discussion presented herein the paper, let us comment on the links of the IMD method with other method used in topology optimization. Comments will be limited to one of the most popular method called SIMP (Solid Isotropic Material with Penalization, see e.g. [1, 17-23]). Using the notation adopted in [18] and changing it slightly to fit to the notation adopted in this work, let us consider a body occupying a domain $\Omega^{\text {mat }} \subset \Omega$ which is part of a larger reference domain $\Omega$, being equivalent to the domain occupied by the three-dimensional elastic body $\Omega$ designed in this paper. In the original formulation of SIMP for isotropic bodies, the optimal design problem consists (similar as in FMD) in searching for optimal fields $\Omega \ni x \rightarrow H_{i j k l}(x)$ rep- 
resenting the components of isotropic Hooke tensor, which are the design variables over the domain $\Omega$. More specifically, the minimum compliance (maximum global stiffness) problem takes the following form: find kinematically admissible displacement field $\boldsymbol{u}^{*} \in V(\Omega)$ along with the admissible isotropic Hooke tensor $\boldsymbol{H}^{*} \in E_{a d}$ minimizing the work of the load expressed by the linear form on $V(\Omega)$

$$
f=f(\boldsymbol{u})=\int_{\Gamma_{1}} \boldsymbol{T} \cdot \boldsymbol{u} \mathrm{d} a
$$

and subject to the equilibrium equation in the weak form

$$
\forall \boldsymbol{v} \in V(\Omega) \quad \int_{\Omega} \boldsymbol{H}^{*} \varepsilon\left(\boldsymbol{u}^{*}\right) \cdot \boldsymbol{\varepsilon}(\boldsymbol{v}) \mathrm{d} x=\int_{\Gamma_{1}} \boldsymbol{T} \cdot \boldsymbol{v} \mathrm{d} a
$$

(see text below equation (2.5), p.3 in [18] and (II.37), (II.38), respectively). In problem (VI.1)-(VI.2), $E_{a d}$ represents the set of admissible Hooke elasticity tensors and various possible definitions of $E_{a d}$ are adopted in various variants of the above optimal design problem. Let

$$
\boldsymbol{H}^{0}=H_{i j k l}^{0} e_{i} \otimes e_{j} \otimes e_{k} \otimes e_{l}
$$

be the Hooke tensor of a given isotropic material and let $\left|\Omega^{\text {mat }}\right|$ denote the volume of the unknown, optimal subset $\Omega^{\text {mat }}$. The set $E_{a d}$ of admissible Hooke elasticity tensors is usually assumed as below:

$$
H_{i j k l}(x)=\mathrm{I}_{\Omega^{m a t}}(x) H_{i j k l}^{0}, \quad x \in \Omega,
$$

where

$$
\begin{aligned}
& \mathrm{I}_{\Omega^{\text {mat }}}: \Omega \rightarrow\{0,1\} ; \mathrm{I}_{\Omega^{\text {mat }}}(x)= \\
& =\left\{\begin{array}{llll}
1 & \text { if } & x \in \Omega^{\text {mat }} & \text { material point } \\
0 & \text { if } & x \in \Omega \backslash \Omega^{\text {mat }} & \text { void (no material point }
\end{array}\right. \\
& \int_{\Omega} \mathrm{I}_{\Omega^{m a t}} \mathrm{~d} x=\left|\Omega^{m a t}\right| \leq V .
\end{aligned}
$$

The choice (VI.4)-(VI.6) of admissible materials means generalized shape optimization. The problem is to place one isotropic material optimally within a given domain, keeping its volume as fixed. This problem is ill-posed and needs relaxation, as thoroughly discussed in Ref. [3]. Upon relaxation by homogenization the problem reduces to finding a porous material of very specific properties of varying density $\rho(x)$. The optimal material is a non-homogeneous composite of moduli corresponding to $3^{r d}$ rank layered microstructures (in $3 \mathrm{D})$ or to $2^{\text {nd }}$ rank microstructures (in 2D). The optimal composite is characterized by elasticity potential $W$ depending on $\rho(x)$. Three methods of simplifying the relation $W=W(\rho)$ have been proposed: a) SIMP, see [1, 17-22], RAMP, see e.g. [18] and c) GRAMP, see [17] (which independently is also a comprehensive source of knowledge about the RAMP method), among which only the latter refers to the exact relaxation by homogenization result, hence deserves special attention. All the mentioned methods have the same aim: to achieve suboptimal solutions to the generalized shape optimization problem, free of composite subdomains, inevitably emerging in the exact solutions to the relaxed problem.

Remark VI.1. The inequality (VI.6) expresses the imposed limit $V$ on the amount of material at our disposal and considerably differs from the isoperimetric condition (II.10) (see also (II.11), (II.21), (II.22), (II.23), (II.24) 2 ) which can in no way be related with the condition (VI.6) limiting the volume $\left|\Omega^{\text {mat }}\right|$ of the material. This property is the first serious obstacle when trying to compare this formulation of topology optimization with the formulation presented earlier and called IMD method.

Moreover, note that the objective function $f=f(\boldsymbol{u})$ is non-smooth with respect to the design variables $H_{i j k l}=$ $H_{i j k l}(x)$, which excludes the possibility of calculating e.g. its gradient. Also, many other numerical problems appear, inter alia, from the disappearance of the material in increasing (during the optimization process) subdomains. This suggests replacing the integer design parameters in problem (VI.4)(VI.6) with continuous design variables along with introducing some form of penalty that steers the continuous solution to discrete $0-1$ values. The most popular and efficient is the aforementioned SIMP method which replaces representation (VI.4) and condition (VI.6) with the following ones:

$$
H_{i j k l}(x)=\rho(x)^{p} H_{i j k l}^{0}, \quad x \in \Omega \quad(p>1)
$$

$$
\int_{\Omega} \rho(x) \mathrm{d} x \leq V, \quad 0 \leq \rho(x) \leq 1, \quad x \in \Omega,
$$

where $\rho=\rho(x)$ is the unknown design function called material density. Function $\rho$ interpolates continuously the material properties between 0 and 1 (i.e. between the void and the material of moduli $H_{i j k l}^{0}$ ) and at the same time evaluates the volume of the body in (VI.8). It turns out that under certain assumptions concerning the condition (VI.8) and for sufficiently large values of the parameter $p>1$, the solution to the SIMP problem tends to stable suboptimal solution to the original 0-1 problem (see e.g. the discussion on pp. 6-7 in [18]). The question of whether it is always possible to construct the microstructure of the material, which would have the same (or almost the same) properties as the properties of the optimal material found by the SIMP method has, inter alia, a close relationship with the Hashin-Shtrikman bounds for two-phase materials and enforces some inequality restrictions on the power $p$ given e.g. in formulae (1.5) on p.7 in [18]. For example, it is suggested to use parameter $p \geq 3$ in $3 \mathrm{D}$ problems for assumed value of Poisson ratio $\nu^{0}=1 / 3$ of the given base material $\boldsymbol{H}^{0}$. On the other hand, the typical use of parameter $p$ with values $p=3$ or $p=5$ in SIMP method results in the designs that "... often converge to local minima, except if some tricky filtering technique is 
used ...", see Remark 5.2.10 on p. 393 in [3]. In the case of the interpolation applied in the SIMP method, the additional lower bound $\rho_{\min }>0$ on the density $\rho$ in (VI.8) has to be introduced

$$
\int_{\Omega} \rho(x) \mathrm{d} x \leq V, \quad 0<\rho_{\min } \leq \rho(x) \leq 1, \quad x \in \Omega
$$

in order to prevent from possible singularity of the equilibrium equation (VI.2) ; in typical applications its value is assumed to be $\rho_{\min } \approx 10^{-3}$.

Remark VI.2. The positive lower bound $\rho_{\text {min }}$ guarantees the existence of the unique displacement field $\boldsymbol{u}$ at each iteration of the SIMP method, because it protects against the ill-conditioning of the square, stiffness matrix $\boldsymbol{K}$ in numerical implementation of the displacement based FEM (algorithm of the SIMP method together with the description of the updating of the density field $\rho=\rho(x)$ is presented on pages 13 and 10 in [18], respectively). On the other hand, in the numerical implementation of the stress based IMD method, the approximation of the stress field $\tau$ is used in variational equilibrium equation (II.5) thus omitting equation (VI.2). The consequence is that the rectangular equilibrium (statics) matrix $\boldsymbol{B}$, does not depend on any design variables unlike the stiffness matrix $\boldsymbol{K}$ in SIMP method, which explicitly depends on the design variable - density $\rho$. The approximation of the statically admissible minimizer $\tau^{*}$ of the approximate functional (III.11) determines explicitly the optimal, nonhomogeneous, isotropic tensor $\boldsymbol{H}^{*}=\boldsymbol{H}\left(\boldsymbol{\tau}^{*}\right)$ (see (II.29), (II.33)). At all points $x \in \Omega$ where the two optimal fields $k^{*}=k^{*}(x), \mu^{*}=\mu^{*}(x)$ reach simultaneously values close or equal to 0 , it is assumed that the optimal material disappears while in the SIMP method we assume that the material disappears if the optimal density field $\rho^{*}=\rho^{*}(x)$ defining the optimal tensor $\boldsymbol{H}^{*}=\boldsymbol{H}\left(\rho^{*}\right)$ reaches values close or equal to the lower permissible value $\rho_{\min }>0$. In the case when the density field $\rho^{*}=\rho^{*}(x)$ reaches values close or equal to the upper permissible value 1 , the material at such points $x \in \Omega^{\text {mat }} \subset \Omega$ exists and its properties are defined by the given base tensor $\boldsymbol{H}^{0}$, see (VI.7). It follows that at points $x \in \Omega$ where the optimal material exists, the compared methods lead to completely different elastic properties because in the IMD method they are defined by both optimal fields $k^{*}(x)$ and $\mu^{*}(x)$ in contrast to the method SIMP in which they are defined by a predetermined base elasticity tensor $\boldsymbol{H}^{0}$. It should be emphasized that at any point $x \in \Omega$ the components of the optimal tensor $\boldsymbol{H}^{*}$ in the IMD method are not upper-bounded in contrast to the SIMP method, in which they can achieve the maximum value resulting from the given base tensor $\boldsymbol{H}^{0}$. The global isoperimetric condition (see (II.10) 2 and (II.21)) of the IMD method implies only integrability of the optimal moduli, which does not mean imposing any upper bounds, which once again highlights the fundamental differences in the interpretation of the conditions (II.10) $)_{2}$ and
(VI.9) in IMD and SIMP method, respectively. Numerical simulations performed by the IMD method (see [14]) confirm the characteristic property of optimal solutions $k^{*}$ and $\mu^{*}$ that almost always vary drastically within the design domain $\Omega$. This behavior of the final results reflects the lack of any conditions in IMD preventing the optimal moduli from achieving certain levels. This is a feature similar to that observed in elasticity problems in which no stress limits are imposed, thus leading to stress singularities around e.g. the re-entrant corners of the domains. To prevent from such solutions and to cut the extremes of the stress plots one should e.g. set the problem as elasto-plastic. By analogy, one can add some extra constraints on the IMD design to arrive at new designs of smaller variations of the optimal moduli. Such conditions have not been imposed in order to show the optimum design free of any additional, technological constraints: the latter can always be assumed, while the pure optimization results seem to be of certain value, like exact elasticity solutions with all their picks of stress concentrations are no less informative than elasto-plastic solutions affected by extra parameters defining the stress limit. Taking advantage of the method presented in [15], more realistic IMD designs could be constructed making them Functionally Graded manufacturable.

On the other hand, another serious problem in the SIMP method is a vague physical interpretation of the solution $\boldsymbol{H}^{*}=\boldsymbol{H}\left(\rho^{*}\right)$ for intermediate values $\rho_{\min } \ll \rho^{*}(x) \ll 1$ of optimal field $\rho^{*}$ (called grey solution), see a comprehensive and thorough discussion from p. 22 in monograph [18].

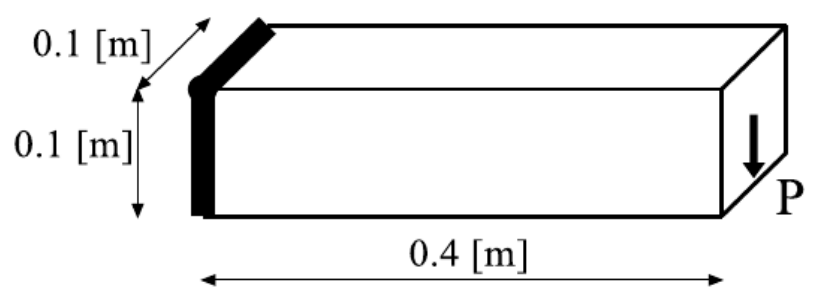

Fig. 2. The body $\Omega$ - cantilever $L_{x} \times L_{y} \times L_{z}=0.4 \times 0.1 \times 0.1[\mathrm{~m}]$ loaded with vertical force $P[N]$

The comments in Remarks VI.1 and VI.2 indicate significant differences in the interpretation of the IMD and SIMP methods. Despite these differences, a visual comparison of the numerical results of the topology optimization for both of these methods is worth showing. For comparison, the two numerical solutions are presented below. In the first example, the $3 \mathrm{D}$ cantilever, supported on the left vertical surface, of length $L_{x}=0.4[\mathrm{~m}]$, width $L_{y}=0.1[\mathrm{~m}]$ and height $L_{z}=0.1[\mathrm{~m}]$ is loaded by the vertical force $P$ applied centrally at the right vertical surface (see Fig. 2). The design domain was subdivided with $64 \times 16 \times 16=16384$ SOLID95 type elements. The structure was loaded with a single force of magnitude $P=100$ [N]. The SIMP solution is implemented by the optimality criteria (OC) method. Volume of the structure is used as a topological constraint, while structural compliance is the 
objective. Tolerance for convergence was set to 0.001 . The final optimal topology found by the ANSYS system (see [23]) is shown in Fig. 3. Not all elements are plotted. These with low pseudo-density (below 0.75 or sometimes more) are hidden, in order to show the most consistent topology. Results are plotted as a contour plot (element solution). The solution is converged after 47 iterations.

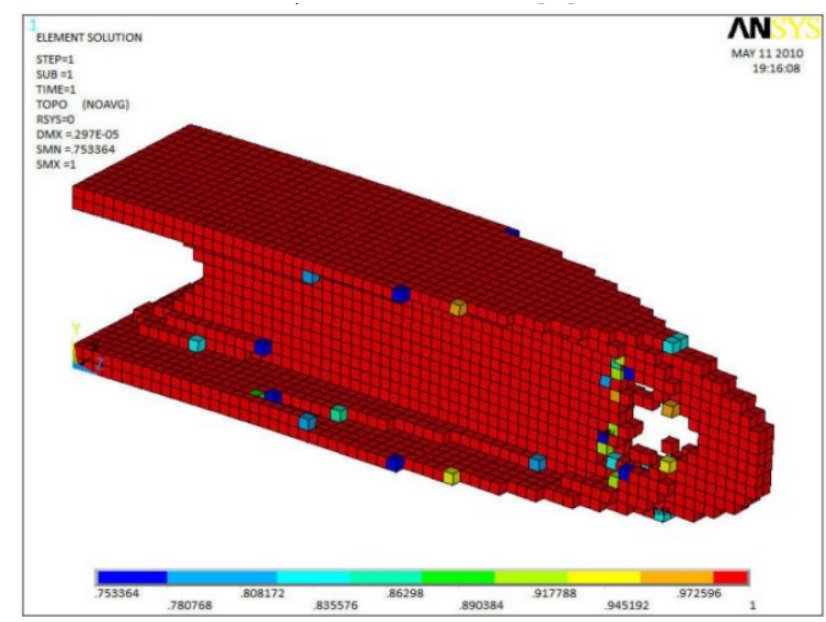

Fig. 3. The optimal topology found by OC method (see [23]) in ANSYS system
The solution is then compared with the FMD and IMD solutions. In Fig. 4, the final topologies represented by the optimal Kelvin modulus $\lambda_{1}^{*}$ found by FMD method (first figure) and final topology represented by the bulk $k^{*}$ and shear $\mu^{*}$ moduli (second and third figure) or equivalently by the Young modulus $E^{*}$ and Poisson ratio $\nu^{*}$ (fourth and fifth figure) found by the IMD method are shown. As in Fig. 3, not all "material points" are plotted. The effect of the "removed material" represented by the very low values of the optimal fields $k^{*}, \mu^{*}$ was shown by the selection of appropriate parameters in Opacity Mapping window available in Voxler graphical system.

In the second example, the 2D cantilever, supported on left vertical side, of length $L_{x}=0.4[\mathrm{~m}]$ and height $L_{y}=0.1[\mathrm{~m}]$ is loaded with the vertical force $P=1.0[\mathrm{~N}]$ applied centrally at the right vertical side (see Fig. 5). The GRAMP interpolation scheme [17] is applied to find the suboptimal layout of basic isotropic material. Results obtained in this way (Fig. 6a) are compared to the distribution of materials ensuing from the homogenization theory (Fig. 6b) as well as those related to the original SIMP (Fig. 6c) and RAMP (Fig. 6d) approach. Young's modulus of an isotropic solid and Poisson's ratio are assumed to be equal $E_{0}=1$ and $\nu_{0}=1 / 3$, respectively. All calculations are coded in MATLAB by G. Dzierżanowski (see [17]) and performed by FEM using the mesh of $400 \times 100$ square, four-node, isoparametric

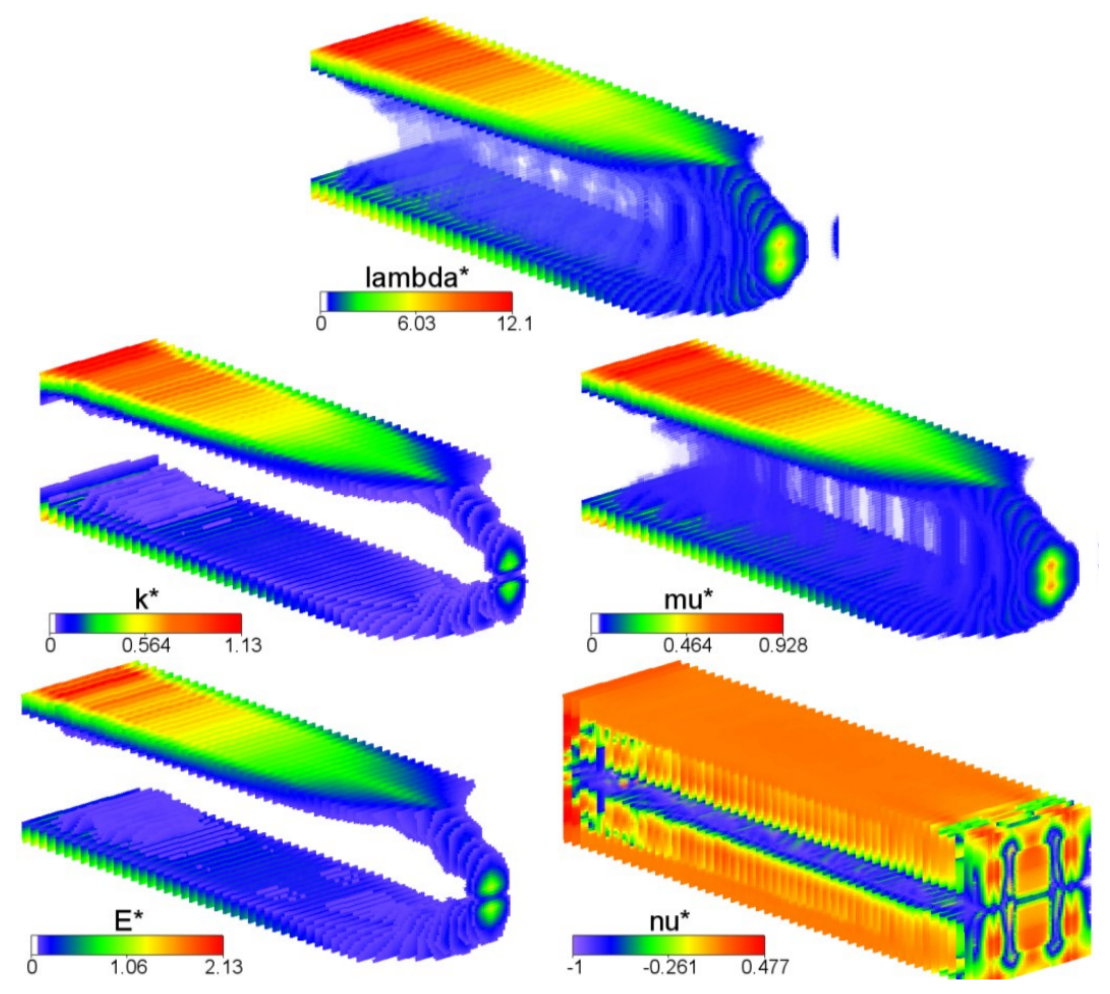

Fig. 4. a) The optimal FMD topology found on the basis of the optimal Kelvin modulus $\lambda_{1}^{*}$, b) optimal topologies due to IMD: the optimal bulk and shear moduli $k^{*}, \mu^{*}$, optimal Young modulus $E^{*}$ and optimal Poisson ration $\nu^{*}$ (first to fifth figure, respectively) 
elements C2D4. It should be emphasized that the optimal layout found by GRAMP method (for $p=1$ ) is in very good agreement with the optimal layout found on the base of homogenization theory and both optimal compliances are almost the same.

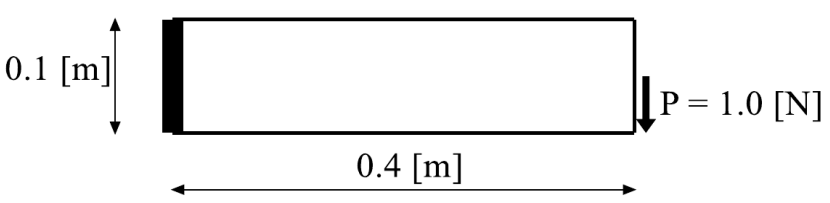

Fig. 5. The body $\Omega$ - cantilever $L_{x} \times L_{y}=0.4 \times 0.1[\mathrm{~m}]$ loaded with vertical force $P=1.0[N]$

Slightly higher values of optimal compliances calculated on the base of RAMP (for $p=2$ ) and SIMP ( $p=3$ ) method, respectively, show that the optimal layouts found by these two methods represent only suboptimal solutions.

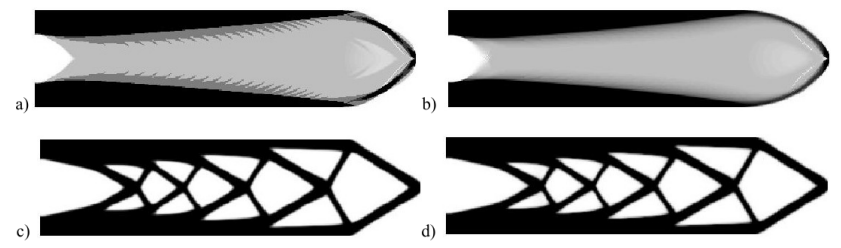

Fig. 6. The optimal topologies found by GRAMP $(p=1)$ method (a), by the homogenization method (b), by SIMP $(p=3)$ method (c) and RAMP ( $p=2)$ method (d) by G. Dzierżanowski program written in MATLAB (see [17])

In Fig. 7, the final topologies represented by the bulk $k^{*}$ and shear $\mu^{*}$ moduli (first and second figure) or equivalently by the Young modulus $E^{*}$ and Poisson ratio $\nu^{*}$ (third and fourth figure) found by IMD method for 2D case are shown. As in Fig. 4, not all "material points" are plotted.

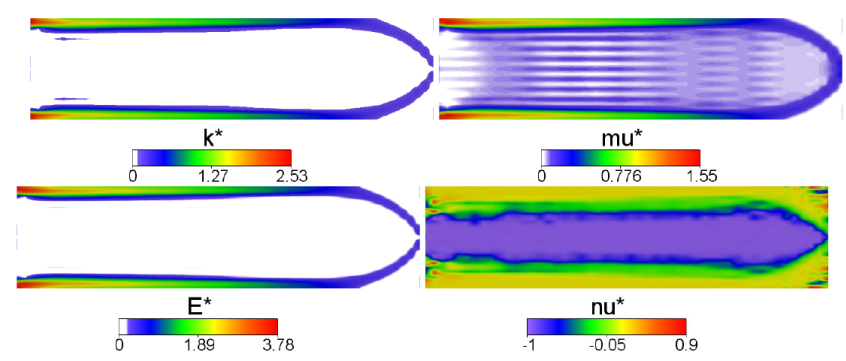

Fig. 7. The final topologies represented by the optimal bulk $k^{*}$ and shear $\mu^{*}$ modulus (first and second figure) or equivalently by the Young modulus $E^{*}$ and Poisson ratio $\nu^{*}$ (third and fourth) found by IMD method for 2D case

A visual evaluation of optimal topologies found on the basis of the SIMP and IMD method can lead to a conclusion that both types of solutions are very similar. Yet one should remember that a) the layout delivered by SIMP is the generalized shape, while the IMD produces the layout of isotropic moduli; b) the IMD method involves shape optimization, since this method indicates the domain where the material should be removed.

\section{Acknowledgements}

The paper was prepared within the Research Grant no 2013/11/B/ST8/04436 financed by the National Science Centre (Poland), entitled: Topology optimization of engineering structures. An approach synthesizing the methods of: free material design, composite design and Michell-like trusses.

This research was carried out with the support of the Interdisciplinary Centre for Mathematical and Computational Modelling (ICM) University of Warsaw under grant no. G506, entitled: Topology Optimization of Structures.

Special thanks to Dr hab. G. Dzierżanowski, who performed numerical calculations of the $2 \mathrm{D}$ cantilever on the base of his original program (see [17]) of finding the optimal composites in plane shape optimization by homogenization, SIMP, RAMP and GRAMP methods.

Special thanks to Szczepaniak, MSc. P., who allowed for the placement of copied figure 3 of optimal solution found in the system ANSYS from his MSc thesis [23].

\section{References}

[1] M.P. Bendsøe, Optimization of Structural Topology, Shape, and Material, Springer, Berlin 1995.

[2] A.V. Cherkaev, Variational methods for Structural Optimization, Springer, New York 2000.

[3] G. Allaire, Shape optimization by the homogenization method, Springer, New York, 2002.

[4] A. Cherkaev, G. Dzierżanowski, Three-phase plane composites of minimal elastic stress energy: High-porosity structures, International Journal of Solids and Structures, 50, 4145-4160 (2013).

[5] M.P. Bendsøe, J.M. Guedes, R.B. Haber, P. Pedersen, J.E. Taylor, An analytical model to predict optimal material properties in the context of optimal structural design, J Appl Mech Trans ASME, 61, 930-937 (1994).

[6] R. Werner, Free material optimization. $\mathrm{PhD}$ thesis, Institute of Applied Mathematics II, University of Erlangen-Nuerenberg, Germany (2000).

[7] J. Haslinger, M. Kočvara, G. Leugering, M. Stingl, Multidisciplinary free material optimization, SIAMJ Appl Math 70, 2709-2728 (2010).

[8] S. Czarnecki, T. Lewiński, A stress-based formulation of the free material design problem with the trace constraint and single loading condition, Bull Pol Acad Sci Tech Ser 60, 191-204 (2012).

[9] S. Czarnecki, T. Lewiński, The free material design in linear elasticity, Topology Optimization in Structural and Continuum Mechanics. CISM International Centre for Mechanical Sciences 549. Courses and Lectures, G.I.N. Rozvany, T. Lewiński (eds.) (Springer Wien Heidelberg New York Dordrecht London, CISM, Udine 2014), 213-257 (2014). 
[10] S. Czarnecki, T. Lewiński, A stress-based formulation of the free material design problem with the trace constraint and multiple load conditions, Struct Multidisc Optim 49(5), 707731 (2014).

[11] A.N. Norris, The isotropic material closest to a given anisotropic material, Journal of Mechanics of Materials and Structures 1, 2 (2006).

[12] G. Duvaut, J.L. Lions, Inequalities in Mechanics and Physics, Springer, Berlin 1976.

[13] K.W. Wojciechowski, Remarks on "Poisson Ratio beyond the Limits of the Elasticity Theory", Journal of the Physical Society of Japan 72(7), 1819-1820 (2003).

[14] S. Czarnecki, P. Wawruch, The emergence of auxetic material as a result of optimal isotropic design, submitted, DOI: $10.1002 /$ pssb.201451733

[15] R. Dwivedi, S. Zekovic, R. Kovacevic, Field feature detection and morphing-based process planning for fabrication of geometries and composition control for functionally graded materials, Proceedings of the Institution of Mechanical Engineers, Part B: Journal of Engineering Manufacture 220, 16471661 (2006).

[16] W.H. Press, S.A. Teukolsky, W.T. Vetterling, B.P. Flannery, Numerical Recipes in C. The Art of Scientific Computing, Cambridge University Press 1992.
[17] G. Dzierżanowski, On the comparison of material interpolation schemes and optimal composite properties in plane shape optimization, Struct Multidisc Optim 46(5), 693-710, 2012.

[18] M.P. Bendsøe, O. Sigmund, Topology Optimization. Theory, Methods and Applications, Springer, Berlin 2003.

[19] R. Kutyłowski, B. Rasiak, Incremental method of Young's modulus updating procedure in topology optimization, $19^{\text {th }}$ International Conference on ComputerMethods in Mechanics, 9-12 May 2011, Warsaw, Poland, 8 pages on CD, 2011.

[20] R. Kutyłowski, M. Szwechłowicz, Special kind of multimaterial topology optimization, Archives of Civil and Mechanical Engineering, Vol. 13, Issue 3, 334-344 (2013).

[21] R. Kutyłowski, B. Rasiak, Application of topology optimization to bridge girder design, Structural Engineering and Mechanics 51(1), 39-66 (2014).

[22] R. Kutyłowski, B. Rasiak, The use of topology optimization in the design of truss and frame bridge girders, Structural Engineering and Mechanics 51(1), 67-88 (2014).

[23] P. Szczepaniak, Topology optimization as a design tool, MSc thesis. Department of Structural Mechanics and Computer Aided Engineering, Warsaw University of Technology and Technical University of Cartagena, Structures and Construction Department, June 2012.

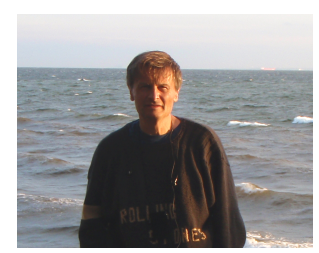

Sławomir Czarnecki. Master's Degree (1983), Warsaw University of Technology, Master's Thesis title: Equations of non-shallow shells spanned over rectangular domains. Ph.D. (1991), Warsaw University of Technology, Faculty of Civil Engineering, Dissertation title: Models of the countable media in the statics of structures. Affiliation: Warsaw University of Technology, Faculty of Civil Engineering, Department of Structural Mechanics and Computer Aided Engineering. Positions held: Assistant Professor. Teaching activity and courses: Theoretical \& Structural Mechanics, Computer Science, Object-Oriented Programming, Finite Element Method. Field of recent research work: Topology \& Structural Optimization, Finite Element Method, Programming in $\mathrm{C} / \mathrm{C}++$. 